\title{
Robotische Chirurgie - operiert der Roboter?
}

\section{Robotic surgery - who is the boss?}

\section{다 (i) (요 $\ominus$}

\author{
Autor
}

Patrick J. Schuler

Institut

Klinik für Hals-Nasen-Ohrenheilkunde, Kopf- und Halschirurgie, Universitätsklinikum Ulm

Schlüsselwörter

Robotik, TORS, Kopf-Hals-Tumor, Haptik, Kosten, Studien

Key words

robotic, TORS, head and neck cancer, costs, clinical studies

\section{Bibliografie}

DOI https://doi.org/10.1055/s-0043-121791

Laryngo-Rhino-Otol 2018; 97: S231-S255

(c) Georg Thieme Verlag KG Stuttgart · New York

ISSN 1615-0007

\section{Korrespondenzadresse}

Prof. Dr. med. Patrick J. Schuler

Klinik für Hals-Nasen-Ohrenheilkunde, Kopf- und

Halschirurgie, Universitätsklinikum Ulm

Frauensteige 12

D-89075 Ulm

Tel.: +49-731-500-59501, Fax:+49-731-500-59502

Patrick.Schuler@uniklinik-ulm.de

\section{ZUSAMMENFASSUNG}

Der Roboter-assistierten Chirurgie (RAC) im Kopf-Hals-Bereich wird ein großes Potenzial zugeschrieben. Vor allem in der operativen Onkologie wird der Einsatz von Roboter-Systemen als besonders interessant erachtet. Bislang sind 2 Gerätetypen (DaVinci ${ }^{\circledR}$ und FLEX ${ }^{\circledR}$ ) für die klinische Anwendung im Kopf-HalsBereich zugelassen, und multiple weitere Systeme befinden sich in der präklinischen Erprobungsphase. Auch wenn prinzipiell bestimmte Patientengruppen von der RAC profitieren könnten, finden sich keine systematischen randomisierten Studien. Ein möglicher Vorteil der RAC gegenüber den bisherigen Standardverfahren kann daher bisher nicht nachgewiesen werden. Der begrenzte klinische Nutzen und die finanzielle Zusatzbelastung scheinen aktuell der Hauptgrund zu sein, warum der flächendeckende Einsatz der RAC bislang ausgeblieben ist. Diese Übersichtsarbeit beschreibt die verschiedenen Anwendungsmöglichkeiten der RAC im Kopf-Hals-Bereich. Außerdem werden die finanziellen und technischen Herausforderungen, sowie anstehende Weiterentwicklungen der RAC beleuchtet. Besonderes Augenmerk wird auf spezielle Risiken der Roboter-Chirurgie und aktuell laufende klinische Studien gelegt. Mittelfristig wird davon ausgegangen, dass die RAC Einzug in die klinische Routine erhalten wird und sich das medizinische Personal zunehmend mit den technischen, wissenschaftlichen und auch ethischen Besonderheiten auseinandersetzen muss.

\section{ABSTRACT}

In the head and neck region, great potential is seen in robotassisted surgery (RAS). Mainly in cancer surgery, the use of robotic systems seems to be of interest. Until today, two robotic systems (DaVinci ${ }^{\circ}$ und FLEX ${ }^{\circledR}$ ) have gained approval for clinical use in the head and neck region, and multiple other systems are currently in pre-clinical testing.

Although, certain groups of patients may benefit from RAS, no unbiased randomized clinical studies are available. Until today, it was not possible to satisfactorily prove any advantage of RAS as compared to standard procedures. The limited clinical benefit and the additional financial burden seem to be the main reasons, why the comprehensive application of RAS has not been realized so far.

This review article describes the large variety of clinical applications for RAS in the head and neck region. In addition, the financial and technical challenges, as well as ongoing developments of RAS are highlighted. Special focus is put on risks associated with RAS and current clinical studies. We believe, that RAS will find its way into clinical routine during the next years. Therefore, medical staff will have to increasingly face the technical, scientific and ethical features of RAS. 


\section{Inhaltsverzeichnis}

\begin{tabular}{lll} 
& Zusammenfassung & $\mathrm{S} 231$ \\
1. & Abstract & $\mathrm{S} 231$ \\
2. & Rinleitung & $\mathrm{S} 232$ \\
3. & Ooboter-Systeme & $\mathrm{S} 232$ \\
4. & Nasopharynx & $\mathrm{S} 233$ \\
5. & Larynx & $\mathrm{S} 235$ \\
6. & Nasennebenhöhlen und Schädelbasis & $\mathrm{S} 236$ \\
7. & Schilddrüse & $\mathrm{S} 237$ \\
8. & Halsweichteile & $\mathrm{S} 239$ \\
9. & Haptik & $\mathrm{S} 240$ \\
10. & Kosten & $\mathrm{S} 242$ \\
11. & Fallzahlen & $\mathrm{S} 243$ \\
12. & Risiken & $\mathrm{S} 245$ \\
13. & Klinische Studien & $\mathrm{S} 245$ \\
14. & Neue Technologien & $\mathrm{S} 247$ \\
15. & Schlussfolgerung & $\mathrm{S} 248$ \\
& Literatur & $\mathrm{S} 249$ \\
& & $\mathrm{~S} 250$ \\
& & \\
\hline & & \\
\hline
\end{tabular}

\section{Einleitung}

Die Roboter-assistierte Chirurgie (RAC) hält zunehmend Einzug in die Kopf-Hals-Chirurgie, ist aber noch vielfach umstritten. Die Vorteile der RAC liegen darin, dass sie unsere operativen Fähigkeiten nicht nur durch Verbesserung unserer traditionellen chirurgischen Methoden verändert, sondern auch die Möglichkeit neuer operativer Zugänge bietet [63]. An fokussierten Zentren wird die RAC regelmäßig für gutartige und maligne Erkrankungen im Oropharynx eingesetzt. Besonders im asiatischen Raum werden auch immer öfter Roboter-assistierte Eingriffe an der Schilddrüse durchgeführt. Dies spiegelt sich sowohl in der Anzahl der verkauften Roboter-Systeme als auch in den Fallzahlen für RAC wider, welche weltweit zunehmen. Die RAC bietet eine optimierte Visualisierung und Erreichbarkeit des Operationsgebiets, wodurch das Gewebetrauma reduziert werden kann. Zusätzlich erscheinen verbesserte ästhetische Resultate möglich, da in einigen Fällen auf eine konventionelle Operation mit offener Schnittführung verzichtet werden kann. Trotz allem hat sich die RAC in Europa auch im Kopf-Hals-Bereich noch nicht vollständig durchgesetzt. Die Gründe hierfür liegen in der aufwändigen Handhabung der Roboter-Systeme und dem finanziellen Mehraufwand bei häufig fehlender Gegenfinanzierung. Vor allem aber zeigt sich bislang klinisch kein sicherer Vorteil der RAC gegenüber konventionellen Operationsverfahren wie der transoralen Lasermikrolaryngoskopie (TLM).

Ein Roboter ist ein programmierbares Mehrzweckwerkzeug, welches für das Bewegen von Material, Werkstücken und Spezialgerä- ten eingesetzt wird. Der Bewegungsablauf ist frei programmierbar, und der Roboter ist daher für verschiedenste Aufgaben einsetzbar [2]. Üblicherweise wird ein Roboter verwendet, um sich wiederholende, ermüdende Tätigkeiten mit hoher Präzision und Reproduzierbarkeit zu übernehmen. Im Allgemeinen wird einem Roboter daher ein hohes Maß an autonomer Tätigkeit zugeschrieben, was auf die in der Medizin eingesetzten Geräte allerdings meistens nicht zutrifft. Die Einschränkungen hierfür sind in ungelösten technischen Herausforderungen und vorliegenden Sicherheitsbedenken zu finden.

Die heute eingesetzten Roboter-Systeme sind Computer-gestützte Operationswerkzeuge, welche im besten Fall die Operationsabläufe optimieren und die Genauigkeit des Eingriffs erhöhen können. Die folgende Übersichtsarbeit beschreibt die neuesten Entwicklungen der RAC und ist nach anatomischen Unterregionen des KopfHals-Bereiches unterteilt. Im Weiteren werden die haptischen Aspekte, die Risiken und die finanzielle Komponente der RAC diskutiert, sowie aktuell laufende klinische Studien vorgestellt.

\section{Roboter-Systeme}

Der Großteil der weltweit durchgeführten Roboter-assistierten Operationen im Kopf-Hals-Bereich wird aktuell mit dem DaVinci-System der Firma Intuitive Surgical (Sunnyvale, USA) durchgeführt ( $>$ Abb. 1). Die Firma ist in den 80er-Jahren aus dem Forschungsinstitut ,SRI International' hervorgegangen. Hinlänglich bekannt ist der militärische Hintergrund der Entwicklung, bei dem mithilfe des Roboters rasche operative Eingriffe im Schlachtfeld ohne direkte Anwesenheit des Operateurs durchgeführt werden sollten. Im Jahr 2003 erfolgte die Vereinigung mit ,Computer Motion', dem Hersteller des früheren ZEUS-Roboter-Systems, welches später vom Markt genommen wurde.

Das aktuelle DaVinci-System besteht aus 3 Instrumentenarmen, die einen starren Schaft und einen beweglichen Endeffektor besitzen. Die Instrumente verlieren nach 15-20 Verwendungszyklen ihre Zulassung und werden elektronisch verriegelt. Die Bildgebung erfolgt über einen vierten Kameraarm, der eine 3-dimensionale Visualisierung ermöglicht. Im Gegensatz zu den meisten anderen Systemen erfolgt der Informationsaustausch zwischen dem Roboter-System und dem Operateur beim DaVinci-System rein elektronisch. Somit ist der Abstand zwischen Patient und Operateur variabel und kann im Extremfall mehrere tausend Kilometer betragen [111]. Der DaVinci-Roboter wird hauptsächlich im gynäkologischen Fachbereich eingesetzt, aber unter anderem auch in der Urologie, Allgemeinchirurgie und Kopf-Hals-Chirurgie.

Das DaVinci-System kam 1999 erstmals in Europa auf den Markt und erhielt 2001 zunächst die FDA-Zulassung für die Abdominalchirurgie. Die Arbeitsgruppe um Weinstein et al. in Philadelphia/USA leistete frühe Pionierarbeit zur Anwendung des DaVinci-Systems im Kopf-Hals-Bereich [61], woraufhin 2009 die Zulassung der ,food and drug administration' (FDA) für die transorale Roboter-assistierte Chirurgie (TORS) erfolgte.

Das initiale DaVinci-Modell wurde 2006 durch eine 3-dimensionale Visualisierung verfeinert (DaVinci-S) [16]. Die 3D-Visualisierung führt zu einer signifikanten Verbesserung des Operationsresultats und wird in der Roboter-assistierten Chirurgie in naher Zukunft wohl Standard sein [184]. Während die nächste Si-Generation des DaVinciSystems von 2009 noch auf einem Wagen neben dem Operations- 
a

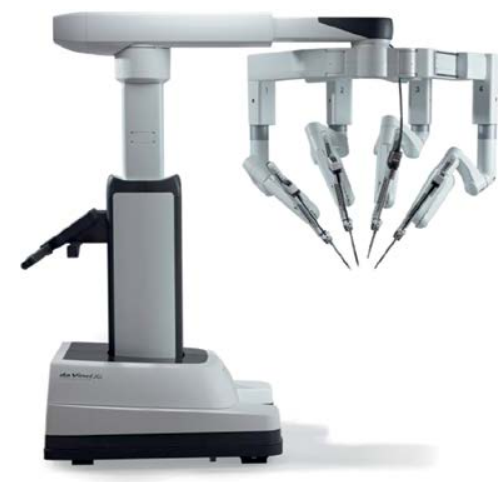

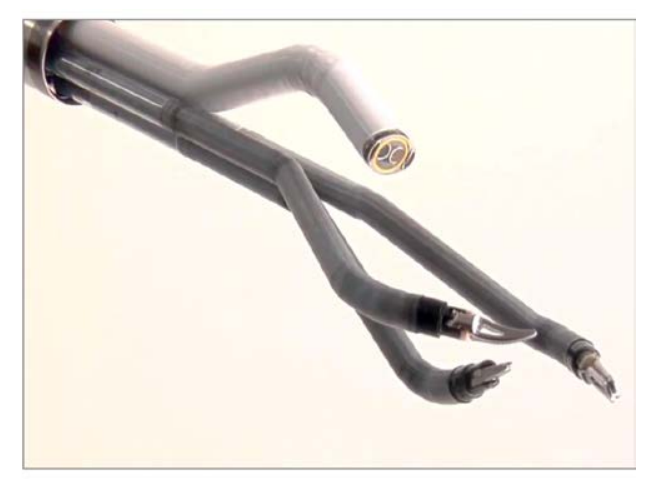

- Abb. 1 Die neuesten Versionen des DaVinci-Systems (Intuitive Surgical, USA). a Die Xi-Generation mit Instrumentenarmen, welche über dem Patienten positioniert werden. b Prototyp des DaVinci-SinglePort Systems mit triangulierbaren Instrumenten. Copyright: 2017 Intuitive Surgical, Inc.

tisch geparkt wird, hat die Xi-Generation von 2014 eine Konstruktion, bei der die Instrumentenarme vollständig über dem Patienten lokalisiert sind ( $>$ Abb. 1a). Die neue, leicht reduzierte X-Generation hat 2017 die CE-Zulassung für den europäischen Markt erhalten. Die X-Generation hat zwar die dünneren Arme des Xi-Systems, ist aber auf einem Beistellwagen wie beim Si-System fixiert. So kann das XSystem mit einem reduzierten Verkaufspreis von ca. 1 Millionen USD auf den Markt gebracht werden, um neue Zielgruppen anzusprechen. Noch keine Zulassung hat das DaVinci-SinglePort (SP)-System erhalten, welches sich aktuell im Rahmen von klinischen Studien in der Erprobung befindet ( $\triangleright$ Abb. 1b).

Neben dem DaVinci-System hat sich nun auch das FLEX ${ }^{\circledR}$-System (Medrobotics, Raynham, USA) für die transorale Roboter-assistierte Chirurgie etablieren können. Die zentrale Komponente des FLEX ${ }^{\circledR}$ System ist ein Computer-gesteuertes flexibles Endoskop, welches sich beim Einführen der nicht-linearen Anatomie des Pharynx angleichen kann ( $\triangleright$ Abb. 2). Somit können prinzipiell auch Patienten behandelt werden, bei denen keine Retroflexion der Halswirbelsäule möglich ist. Nach Arretieren des Endoskops werden anschließend flexible Instrumente durch zusätzliche Arbeitskanäle entlang des Endoskops eingeführt, mit denen der operative Eingriff im Halsbereich durchgeführt werden kann [158]. Die Visualisierung erfolgt über eine HD-Kamera an der Spitze des Endoskops. Die Zulassungsstudie erfolgte an $4 \mathrm{HNO}$-Zentren in Europa mit insgesamt 80 Patienten und führte 2016 zur Zulassung durch die FDA für den amerikanischen Markt [94]. Somit steht mit dem FLEX ${ }^{\circledR}$-System neben dem DaVinci ein weiteres praxistaugliches chirurgisches Assistenzsystem für die Kopf-Hals-Chirurgie zur Verfügung.

\section{Oropharynx}

Das Kerngebiet der transoralen Roboter-assistierten Chirurgie liegt in den Pathologien des Oropharynx inklusive der Tonsillen, des Zungengrundes, sowie der Pharynx-Seitenwände ( $\mathbf{A} \mathbf{A b}$. $\mathbf{3}$ ). Dies ist v. a. in der guten Erreichbarkeit aber auch an der relativ hohen Patientenzahl, welche Erkrankungen in dieser Region aufweisen, begründet. Nachdem das DaVinci-System im Vorfeld für Eingriffe in der Gynäkologie und Urologie zugelassen worden war, konnten Weinstein et al.
2007 erstmals bei 3 Patienten eine Roboter-assistierte supraglottische Laryngektomie dokumentieren [193]. Im selben Jahr erfolgte die Publikation einer prospektiven, einarmigen Phase I Studie bei Patienten mit Tonsillen-Karzinom (T1-2, n=27). Die Resektionsränder waren bei 25/27 Patienten ausreichend, die Komplikationen beinhalteten Schleimhautblutung, Delirium, ungeplante Tracheostomie bei OSAS, leichter Trismus und Näseln [194]. Einige Jahre später folgte die Publikation einer amerikanischen Multicenterstudie mit 192 Patienten, hauptsächlich mit Oropharynx-Karzinomen [192]. Aufgrund der unzureichenden Einstellbarkeit konnte der Eingriff allerdings in 15/192 Patienten ( 7,8\%) nicht vollständig durchgeführt werden.

Basierend auf diesen Machbarkeitsstudien werden heute kleinere Oropharynx-Karzinome (T1-T2) an einigen Zentren routinemäßig mit TORS behandelt [70]. Außerdem konnte seitdem eine Reihe von weiteren klinischen Zentren ihre Erfahrungen mit TORS im Oropharynx beschreiben. Hierzu zählen unter anderem deutsche Arbeitsgruppen ( $n=50, n=17$ ) [109, 113], das französische TORS-Konsortium ( $n=169)$ [8], eine südkoreanische Arbeitsgruppe ( $n=27)$ [103] und eine belgische Multicenterstudie $(n=86)$ [121]. Das Gesamtüberleben in diesen Studien ist regelmäßig vergleichbar mit dem nach primärer Radiochemotherapie [197]. Da es sich bei den TORS-Fällen jeweils um nicht-randomisierte Fallbeschreibungen handelt, muss allerdings von einem deutlichen Bias bei der Patientenselektion ausgegangen werden.

Mit Zunahme der TORS-spezifischen Kenntnisse wurde auch der Parapharyngeal-Raum in die Liste der möglichen Indikation für TORS aufgenommen. So konnten O'Malley et al. eine Fallserie von $10 \mathrm{~Pa}$ tienten mit benignen Tumoren des Parapharyngeal-Raums erfolgreich mit dem DaVinci-System operieren [129]. Ebenso war dies Arshad et al. in 3 Patienten mit benignen oder malignen Tumoren gelungen [7]. Allerdings setzt der TORS-Eingriff in dieser Region eine detaillierte Kenntnis der parapharyngealen Anatomie voraus. Besonders der Verlauf der A. carotis interna und des Nervus glossopharyngeus ist bei transoraler Sichtweise von Bedeutung und wird von Wang et al. im Detail beschrieben [185]. Weiterhin ist die Inzidenz der Pathologien im parapharyngealen Raum gering und nur ein Teil davon kann tatsächlich chirurgisch therapiert werden. 
a

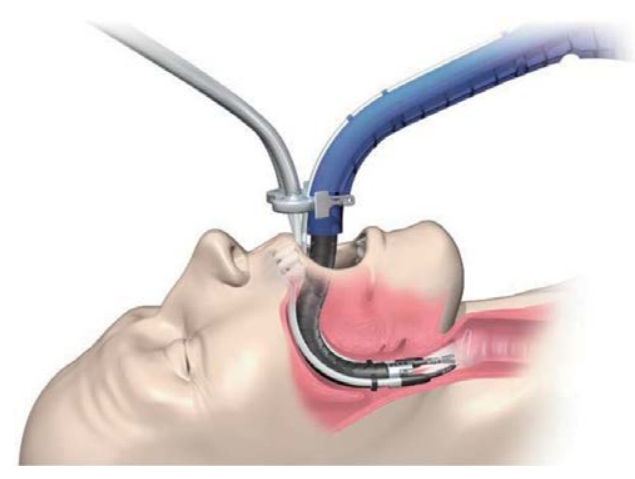

c

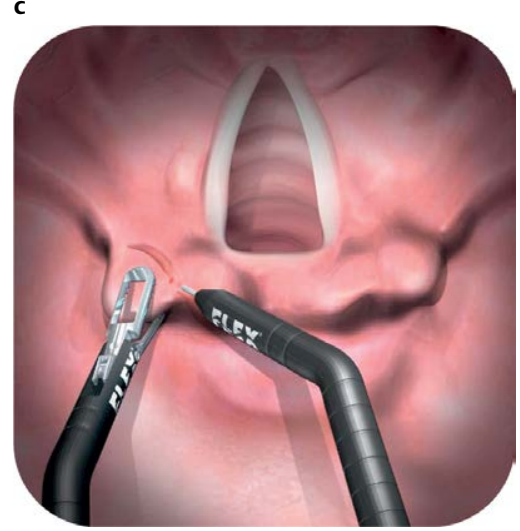

b

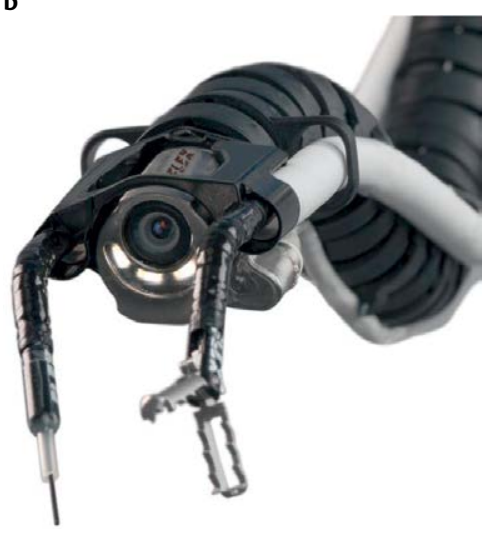

- Abb. 2 Das FLEX ${ }^{\circledR}$ System (Medrobotics, USA). a Der flexible Aufbau ermöglicht die Anpassung an die menschliche Anatomie. $\mathbf{b}$ In der Spitze des Endoskops sind die Kamera und die Lichtquellen integriert. Die flexiblen Instrumente werden über separate Kanäle eingeführt. c Das FLEX ${ }^{\circledR}$ System hat die Zulassung für Eingriffe am Pharynx und Larynx erhalten.

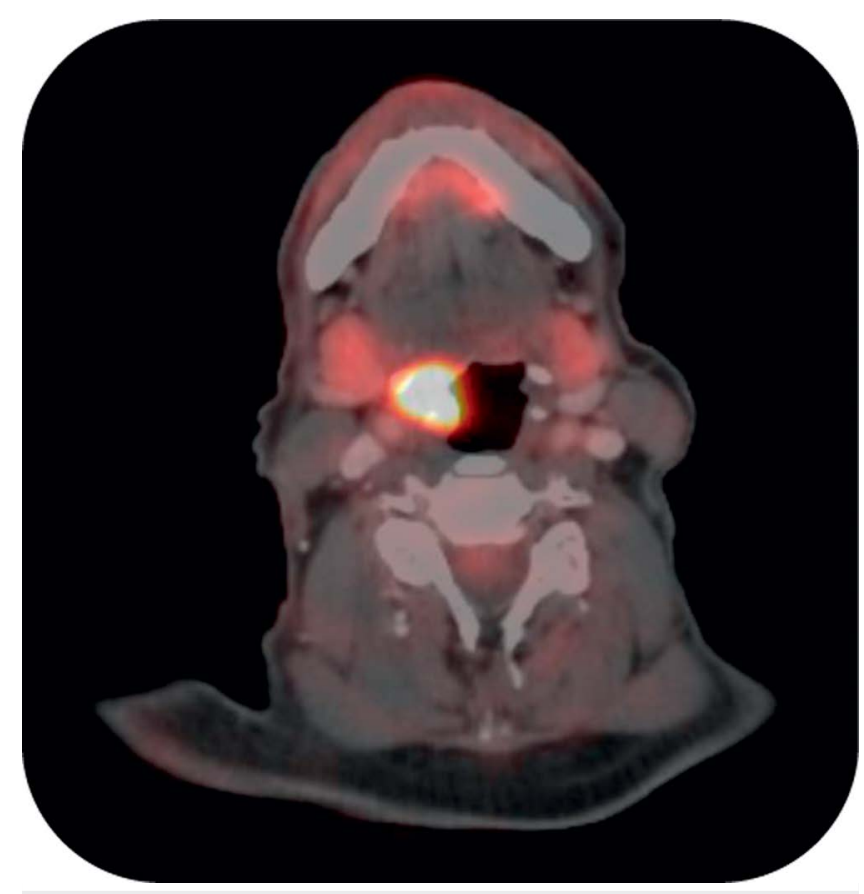

Abb. 3 Die Hauptindikation für TORS sind Tumoren des Oropharynx (T1-2) wie in diesem PET-CT dargestellt. Copyright: Schuler PJ.
Eine besondere Position nehmen Patienten mit obstruktivem Schlafapnoe-Syndrom (OSAS) ein. Da es sich hierbei um eine nichtmaligne Erkrankung mit hoher Inzidenz handelt, wird die Frage, ob sich der finanzielle Aufwand eines Roboter-assistierten Eingriffs rechtfertigen lässt, immer wieder leidenschaftlich diskutiert. Eine Tonsillektomie und Zungengrund-Reduktion kann sicherlich auch ohne Roboter-System adäquat durchgeführt werden. Die Vorteile von TORS werden hingegen mit einem verminderten Operationstrauma und schnellerer postoperativer Heilungsphase angegeben. Ohne Zweifel ist, wie von Vicini et al. beschrieben, die Uvulopalatopharyngoplastik (UPPP) bei korrekter Indikation eine gute Möglichkeit, um die Beschwerden der OSAS-Patienten zu reduzieren ( $n=234)$ [183]. Diese Erkenntnis wird durch die Beobachtungen von Hoff et al. unterstützt $(n=285)$ [62]. Friedman et al. konnten beim Behandlungserfolg einen Vorteil von TORS gegenüber alternativen Operationsmethoden wie Radiofrequenz-Ablation oder submuköse Exzision des Zungengrundes errechnen. Allerdings war die Morbidität in Bezug auf Dysphagie und Heilungsdauer in der TORS-Gruppe signifikant erhöht $(n=27)$ [49]. Golbin et al. konnten aufzeigen, dass die präoperative Schlafendoskopie als Teil der Indikationsentscheidung wesentlich zum Operationserfolg beiträgt [58]. In ihrem Patientenkollektiv ( $n=104$ ) zeigte sich aber auch, dass TORSEingriffe im Vergleich zu konventionellen Operationen einen erhöhten Kostenaufwand und längere Krankenhausliegezeiten verursa- 
chen, ohne dass das operative Ergebnis verbessert werden konnte. Abschließend konnten verschiedene Meta-Analysen den Behandlungserfolg von TORS bei OSAS auch bei übergewichtigen Patienten bestätigen $[55,116]$. Die Frage, ob TORS gegenüber alternativen Operationsmethoden einen Vorteil erbringt, welcher den hohen finanziellen Mehraufwand rechtfertigt, bleibt aber weiterhin unbeantwortet.

Auffallend wenige Arbeitsgruppen haben sich in der Literatur mit der Roboter-assistierten Resektion von Zungengrund-Tumoren beschäftigt. Die Erstbeschreibung erfolgte durch O'Malley et al. 2006 bei 3 Patienten [131]. Eine weitere Fallserie mit 13 Patienten wurde 2013 durch die italienische Arbeitsgruppe um Mercante et al. publiziert [119]. Beide Studien bescheinigten eine gute Durchführbarkeit mit günstigen funktionellen Ergebnissen.

Mit zunehmender Erfahrung der Operateure erscheinen nun auch Roboter-assistierte Eingriffe am Hypopharynx sicher durchführbar. Park et al. publizierten schon 2013 eine retrospektive Studie zum Hypopharynx-Karzinom, in der TORS ( $n=30)$ mit offener Chirurgie verglichen wurde [140]. Unter Berücksichtigung der Selektionsbias zeigte die TORS-Gruppe bessere Werte für die Dekanülierungsrate, Schluckvermögen und Krankenhausliegezeit. Die 5-Jahres-Überlebensrate war für beide Gruppen vergleichbar [141]. Kleinere Fallserien bestätigen die Durchführbarkeit von TORS im Hypopharynx $(n=5)$ und speziell im Sinus piriformis $(n=10)[108,187]$. Die oben beschriebenen Eingriffe wurden jeweils mit den elektrischen Resektionsinstrumenten des DaVinci-Systems durchgeführt. Kucur et al. zeigten beispielhaft an einem Patienten, dass auch im Hypopharynx eine Roboter-assistierte Laserresektion möglich ist [88]. Die flexible Laserfaser wurde hierbei mit dem Nadelhalter geführt. Trotz dieser ermutigenden Beschreibungen, sollte klar bleiben, dass die Patienten für TORS eine optimale Einstellbarkeit des Hypopharynx haben müssen und somit selbstverständlich auch einer transoralen Lasermikrochirurgie zugänglich sind. Eine weitere Patientengruppe, welche potentiell eine Anwendungserweiterung für TORS darstellt, sind die Divertikel im oberen Hypopharynx. Bei Anwendung eines flexiblen Roboter-Systems könnten sich hier Vorteile bei schlecht einstellbaren Patienten ergeben, was bisher in Kadaverstudien demonstriert worden ist [51].

Ein wichtiger Aspekt bei der Auswahl der Therapieoptionen sind die Langzeitverläufe der Patientengruppen. Verschiedene Arbeitsgruppen konnten den TORS-Patienten günstige Überlebensraten und gute funktionelle Ergebnisse bescheinigen [38, 122]. Allerdings sind die chirurgischen und nicht-chirurgischen Behandlungsprotokolle mit denen TORS retrospektiv verglichen wurde, nicht näher beschrieben worden. Ebenso war die Lebensqualität der TORS-Patienten im Langzeitverlauf zufriedenstellend, während eine eventuelle adjuvante Radio-(chemo)-Therapie die Lebensqualität tendenziell reduzierte $[45,66]$.

Eine kleine Gruppe an TORS-Operateuren hat sich in der Vergangenheit mit der Roboter-assistierten Chirurgie von freien Gewebstransplantaten beschäftigt [40]. Die Erstbeschreibung erfolgte 2009 durch Mukhija et al., welche in 2 Patienten ein Radialis-Transplantat Roboter-assistiert zur Pharynx-Rekonstruktion einnähen konnten [125]. Weitere Arbeitsgruppen folgten unter Verwendung von Radialis-, Oberschenkel- oder Jejunum-Transplantaten [18, 31, 56, 142]. In einer weiteren Fallserie wurde ebenfalls ein Radialis-Transplantat zur Defektdeckung nach TORS verwendet, welches aber konventio- nell ohne Roboter-Assistenz eingenäht wurde [13]. Hervorzuheben ist die Publikation von Song et al., in der die Roboter-assistierte Naht einer Gefäßanastomose unter Verwendung spezieller Mikroinstrumente beschrieben wird [167].

\section{Nasopharynx}

Der Nasopharynx bietet sich aufgrund seiner anatomischen Lage potentiell sehr gut für die Roboter-assistierte Chirurgie an. Die traditionelle, offene Chirurgie ist aufwendig und oft nur durch große Zugangswege, wie die Unterkieferspaltung mit entsprechender Morbidität erreichbar [188]. Auf der anderen Seite ist der Nasopharynx mit geeigneten Instrumenten sowohl transnasal als auch transoral durch existierende Körperöffnungen erreichbar, ohne dass weitere Hautinzisionen notwendig werden. Beide Zugänge werden in alleiniger Anwendung oder in deren Kombination im Folgenden diskutiert. Hierbei ergeben sich immer wieder Überschneidungen mit den Zugängen zur Schädelbasis, so dass zusätzlich auf das folgende Kapitel „Nasennebenhöhlen und Schädelbasis“ verwiesen wird. Eine vollständige Roboter-assistierte Nasopharyngektomie wurde erstmalig 2008 durch Ozer et al. im Kadavermodell beschrieben [137]. Die Arbeitsgruppe wählte hierfür den alleinigen transoralen Zugangsweg mit dem DaVinci-System. Die Visualisierung wurde durch eine leichte Kopf-Extension in der Trendelenburg-Position nochmals verbessert, wodurch der gesamte Nasopharynx eingesehen wurde und auch die A. carotis interna bds. unter Sicht präpariert werden konnte. Allerdings war für den Eingriff eine paramediane Gaumenspaltung notwendig.

Eine weiter lateral durchgeführte Gaumenspaltung mit Bildung eines gestielten Weichteiltransplantates wurde durch Tsang et al. beschrieben [179]. Durch die weitere Eröffnung des Gaumens ergibt sich eine bessere Übersicht des Operationsgebiets besonders bei lateral gelegenen Pathologien. Die Wunde kann nach Abschluss der Operation vollständig mit dem Roboter vernäht werden. Die Gaumenspaltung an sich ist nicht wünschenswert, weshalb weitere Systeme für die Roboter-assistierte Chirurgie am Nasopharynx getestet wurden. So konnten Richmon et al. 2014 erstmalig die transorale Nasopharyngektomie mit dem FLEX ${ }^{\circledR}$-System in einer Kadaverstudie beschreiben [147]. Aufgrund der flexiblen Bauweise des computer-gesteuerten Endoskops kann bei gleichbleibender Visualisierung auf die Gaumenspaltung verzichtet werden. Ebenso zeigte das selbe System im Vergleich mit der starren transnasalen Endoskopie Vorteile im Nasopharynx, da 2 Instrumente zeitgleich zum Einsatz kommen [159]. Vielversprechend ist die Beschreibung von Holsinger et al., welche die Möglichkeit hatten, das neue DaVinci-SinglePort System für die Nasopharynx-Chirurgie zu testen [180]. Auch hier konnte im Kadavermodell die vollständige Nasopharyngektomie mit Darstellung der A. carotis interna und des Trigeminus-Nervens ohne Gaumenspaltung durchgeführt werden. Ob der Einsatz eines dritten Instrumentenarms, wie von den Autoren beschrieben, in der Praxis von Vorteil ist, bleibt abzuwarten.

Der rein transnasale, endoskopische Zugang zum Nasopharynx ist besonders für Rezidive von Nasopharynx-Karzinomen im Rahmen der Salvage-Chirurgie mehrfach als „extended endonasal approach“ (EEA) beschrieben worden [29]. Wie von Castelnuovo et al. an $36 \mathrm{~Pa}$ tienten demonstriert, ist dieser Eingriff durch erfahrene Chirurgen durchaus auch bei fortgeschrittenen Karzinomen durchführbar. Auf- 
grund der eingeschränkten Visualisierung und Erreichbarkeit von anatomischen Strukturen, ist dieser Zugang bisher aber nur für ausgewählte Patienten praktikabel. Die Möglichkeit, eine navigierte Biopsie des Nasopharynx mithilfe eines neuartigen Kontinuums-Roboters durchzuführen, ist 2017 von Wu et al. in einem Kadavermodell beschrieben worden [196]. Das System besteht aus 2 konzentrischen, starren Röhren, welche unabhängig voneinander gesteuert werden können. Dieser nicht-lineare Zugang ist vergleichsweise atraumatisch und könnte somit potentiell auch unter Lokalanästhesie zum Einsatz kommen.

Analog zur Roboter-assistierten Schädelbasis-Chirurgie sind auch für Eingriffe am Nasopharynx transorale Zugänge in Kombination mit transantralen oder transzervikalen Zugängen beschrieben worden $[33,36]$. Beide Zugänge ermöglichen eine gute Visualisierung und Manipulation des gesamten Nasopharynx. Aufgrund der deutlich erhöhten Zugangsmorbidität und den fehlenden Bohrinstrumenten beim erweiterten transantralen Zugang sind diese Prozeduren allerdings bisher ausschließlich in Kadaverstudien beschrieben worden.

In der Chirurgie des Nasopharynx ist der kombinierte transnasale/transorale Zugang in der Entwicklung am weitesten fortgeschritten. So führten Dallan et al. 2012 eine vollständige transorale Nasopharyngektomie mit dem DaVinci-System durch [36]. Auf eine Gaumenspaltung konnte verzichtet werden, wenn der Kamera-Arm transnasal eingeführt wurde. Hierfür zeigte sich eine posteriore Septumresektion als hilfreich, um die Visualisierung des gesamten Operationsgebiets zu optimieren. Sinnvoll erscheint auch die Kombination des transoralen Roboter-assistierten Zugangs (TORS) mit der transnasalen endoskopischen Operation. Diese Operationsweise nutzt die Vorteile beider Zugänge, welches sich im Nasopharynx optimal ergänzen können. So konnten Sreenath et al. an drei klinischen Fällen demonstrieren, dass dieses Verfahren prinzipiell durchführbar ist [168]. Die behandelten Patienten litten unter einer retropharyngealen Metastase eines Schilddrüsen-Karzinoms, einem Schleimhautmelanom des Nasopharynx und einer nasopharyngealen Synechie nach nasalem Drogenabusus.

Die Arbeitsgruppe von Wei et al. in Hong Kong besitzt die weitaus größte klinische Erfahrung mit Roboter-assistierten Operationen im Nasopharynx. Bereits 2010 konnte ein Patient mit Rezidiv eines Nasopharynx-Karzinoms transoral mit dem DaVinci-System erfolgreich operiert werden [189]. Trotz Anwendung der Instrumente mit EndoWrist ${ }^{\circledR}$-Design, welche eine erweiterte Rotation im Endbereich erlauben, war wie oben beschrieben für den Eingriff eine Gaumenspaltung notwendig. 2 Jahre später erfolgte die Behandlung eines Patienten, welcher ebenfalls ein Karzinom-Rezidiv am Dach des $\mathrm{Na}$ sopharynx präsentierte [198]. Der Eingriff erfolgte ähnlich wie bei Sreenath et al. mit einem kombinierten transoralen Roboter-assistierten und einem transnasalen endoskopischen Eingriff ohne Roboter-Assistenz, so dass der Tumor en bloc mit ausreichendem Sicherheitsabstand entfernt werden konnte.

Eine Fallserie von 12 Patienten mit Rezidiv eines NasopharynxKarzinoms wurde schließlich 2014 von Tsang et al. publiziert [181]. Das Tumorstadium umfasste T1-T4, und alle Patienten wurden über einen transoralen Zugang und Gaumenspaltung mit dem DaVinciSystem operiert. Bei 11/12 Patienten wurde ein ausreichender Sicherheitsabstand oder eine close-margin Situation erreicht. Die beschriebenen Komplikationen umfassten eine Osteonekrose des Clivus, eine persistierende Gaumen-Fistel und einen hypoxischen
Hirnschaden. In der onkologischen Nachsorge zeigten 2/12 Patienten ein Lokalrezidiv und ein Patient distale Metastasen. Nach Angaben der Autoren lag die Überlebensrate ohne adjuvante Therapie nach 2 Jahren bei $83 \%$. So stellt TORS besonders bei Lokalrezidiven eines Nasopharynx-Karzinoms durchaus eine therapeutische Option mit akzeptablen Ansprechraten dar. Die Arbeitsgruppe diskutiert darüber hinaus den Stellenwert der chirurgischen Therapie bei TumorRezidiven im Nasopharynx im Vergleich zu alternativen Behandlungsoptionen [190]. Für den europäischen Raum wurde aufgrund der geringeren Inzidenz des Nasopharynx-Karzinoms allerdings in einer Meta-Analyse noch eine zurückhaltende Empfehlung für Roboterassistierte Eingriffe am Nasopharynx gegeben [120].

\section{Larynx}

Die konsequente Weiterentwicklung von TORS mündet in den Roboter-assistierten Eingriffen am Larynx. Multiple Publikationen beschreiben Fallserien von Patienten mit supraglottischen Pathologien, was durch die gute Erreichbarkeit der Epiglottis zu erklären ist. Dies steht allerdings in keiner Relation zu der niedrigen Inzidenz von Epiglottis-Karzinomen. Zusätzlich sind diese oft aufgrund der Ausdehnung oder des Patientenalters einer operativen Therapie nicht weiter zugänglich, was sich auch in der niedrigen Fallzahl in den publizierten Fallserien widerspiegelt. Ebenso wie die Transorale LaserMikrochirurgie (TLM) benötigen die starren Instrumente des DaVinci-Systems auch einen weitgehend geradlinigen Zugangsweg (,straight line of sight'), und die Größe des Gerätes führt besonders bei der Larynxchirurgie häufiger zu mechanischen Interferenzen zwischen den Instrumenten. Um die Vorteile von TORS gegenüber der TLM aufzuzeigen, wären auch hier randomisierte klinische Studien notwendig, welche zum aktuellen Zeitpunkt noch nicht vorliegen.

Die wichtigsten Fallserien beinhalten die Erstbeschreibung im Tiermodell und von 3 klinischen Fällen 2007 durch Weinstein et al. $[191,193]$. Das größte Patientenkollektiv liefert die Subgruppenanalyse des französischen TORS-Konsortiums ( $n=84)$ [145]. In dieser Studie sind allerdings die hohen Quoten an unzureichenden Resektionsrändern (52\%) und an Nachblutungen (18\%) überraschend, welche sich nicht mit den Daten anderer Publikationen decken. Solares et al. konnten den Einsatz einer flexiblen $\mathrm{CO}_{2}$-Laserfaser an der Supraglottis beschreiben [164]. Die koreanische Arbeitsgruppe um Kim et al. publizierte deutlich bessere funktionelle Ergebnisse für TORS als für die offene Kehlkopf-Teilresektion bei supraglottischen Karzinomen in einer nicht-randomisierten, prospektiven Studie $(n=34)$, welche unter Rücksicht auf die Selektionsbias interpretiert werden müssen [139]. Weitere Fallserien wurden mit ähnlichen Erkenntnissen publiziert $[118,135]$.

Neben dem DaVinci-System wird auch das FLEX ${ }^{\circledR}$-System für Eingriffe an der Supraglottis eingesetzt. So wurden im Rahmen der europäischen Zulassungsstudie insgesamt 16 Patienten mit supraglottischen Pathologien an der Epiglottis, den Taschenfalten und den Stellknorpeln mit flexibler TORS behandelt [94]. Unabhängig vom System ergibt sich aber bisher kein effektiver Vorteil von TORS gegenüber der in Europa standardmäßig eingesetzten TLM für die supraglottische Larynx-Chirurgie. Hier ist eine speziell auf die LarynxChirurgie abgestimmte Weiterentwicklung der RAC notwendig.

Eine vollständige ,transorale Roboter-assistierte Laryngektomie‘ (TORS-LE) könnte aufgrund seiner minimalen Hautinzision das Risi- 
ko einer Speichelfistel reduzieren, was besonders bei Salvage-Operationen von Vorteil sein kann [54]. Möglicherweise kann so die empfohlene zusätzliche Defektdeckung mittels gestieltem PectoralisLappen bei Salvage-Laryngektomie überflüssig werden [53]. Die Neck dissection kann entweder 2-zeitig durchgeführt oder durch klinische Kontrollen ersetzt werden [47]. Die TORS-LE wird von kaudal über einen kleinen Tracheostomie-Hautschnitt nach kranial präpariert und anschließend von transoral mittels Roboter-System vervollständigt. Die notwendige Pharynx-Naht kann, wie von Lawson et al. beschrieben, ebenfalls von transoral durchgeführt werden [95]. Anschließend folgten amerikanische, australische und chinesische Fallserien mit kleinerer Fallzahl, welche die Durchführbarkeit im klinischen Bereich bestätigten [31, 85, 163]. Für die TORS-LE können außer dem DaVinci-System auch weitere Instrumente verwendet werden. So demonstrierten Fernandez et al. den Einsatz eines Ultraschall-Skalpells für den transoralen Teil der TORS-LE [48]. Unsere Arbeitsgruppe konnte in einem Kadavermodell die Verwendung des FLEX ${ }^{\circledR}$-Systems untersuchen, wobei der transzervikal nicht-robotisch durchgeführte Anteil der Operation deutlich ausgeweitet werden kann [160].

Obwohl das DaVinci ${ }^{\circledR}$-System nicht für den Einsatz an den Stimmlippen entwickelt wurde, häufen sich die Erfahrungsberichte in diesem Bereich. So konnten Blanco et al. 2011 erstmals die Resektion eines T1-Larynx-Karzinoms mit einer flexiblen $\mathrm{CO}_{2}$-Faser publizieren [15]. Im folgenden Jahr berichteten Kayhan et al. über eine Fallserie mit 10 Patienten, bei denen eine Roboter-assistierte Chordektomie durchgeführt wurde [74]. Eine Tracheostomie war nur in einem Fall indiziert, und die Autoren sahen einen Vorteil von TORS gegenüber TLM in Bezug auf Visualisierung und Zugang zum Operationsgebiet. Ähnliche Ergebnisse wurden durch Lallemant et al. beschrieben [93]. Ein Lokalrezidiv wurde bei 2/13 Patienten in der vorderen Kommissur festgestellt, was zeigt, dass auch bei TORS dieser Bereich aufgrund seiner schlechten Exposition als besonders kritisch einzustufen ist. Schließlich konnten Wang et al. eine Fallserie mit glottischen Larynx-Karzinomen beschreiben, welche keine Tracheostomie benötigten und bei einer durchschnittlichen Nachbeobachtungszeit von 40 Monaten kein Lokalrezidiv entwickelt haben $(n=8)$ [186].

Nach entsprechenden Kadaverstudien konnten klinische Eingriffe auf Glottis-Ebene auch mit dem FLEX ${ }^{\circledR}$-System im Rahmen der Zulassungsstudie durchgeführt werden [50, 94]. Da das FLEX ${ }^{\circledR}$-System aber nicht primär für Glottis-Eingriffe konstruiert worden ist, war die Visualisierung in 3 von 5 Fällen frustran, und der Eingriff musste zu

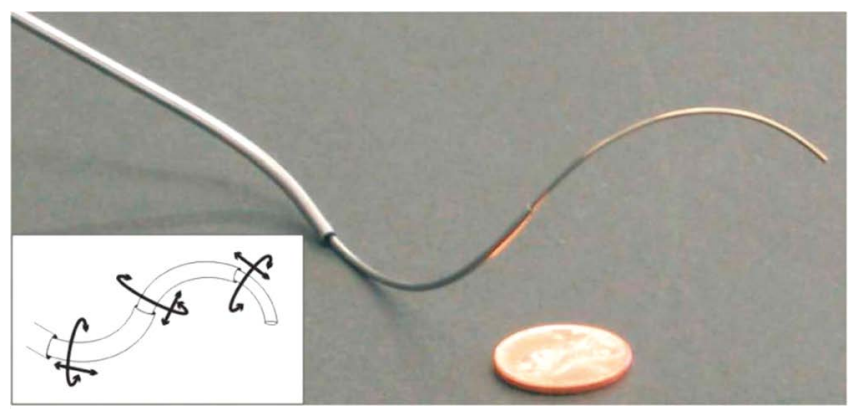

Abb. 4 Der Kontinuums-Roboter des Lehrstuhls für KontinuumsRobotik an der Leibniz Universität Hannover besteht aus konzentrischen Röhren, welche unabhängig voneinander bewegt werden können. einer konventionellen TLM konvertiert werden. Abhilfe könnten neue, kleinere Instrumente schaffen, welche speziell für die LarynxChirurgie entwickelt werden und für 2018 angekündigt sind.

\section{Nasennebenhöhlen und Schädelbasis}

Der Roboter-assistierte Zugang zur Schädelbasis würde die operativen Möglichkeiten an den teils vulnerablen anatomischen Strukturen deutlich erweitern. Aufgrund der technischen Limitationen ist der Einsatz von Roboter-Systemen in diesem Bereich allerdings bisher auf Kadavermodelle und klinische Einzelfallbeschreibungen beschränkt $[171,177]$. Das DaVinci-System erscheint aufgrund seiner Größe nicht die optimale Struktur für den Zugang zur Schädelbasis zu besitzen [155]. Daher existieren mehrere alternative Systeme, welche die speziellen Anforderungen für dieses Operationsgebiet adressieren.

Burgner et al. haben einen Kontinuums-Roboter entwickelt, der transnasal ohne weitere Hilfsschnitte eingeführt wird [22]. Aufgrund seiner Bauweise mit 3 konzentrischen Metallrohren kann über einen Joystick jeder beliebige Punkt in einem 3-dimensionalen Raum nichtlinear angesteuert werden ( $\$$ Abb. 4). Jedes Metallrohr wird über einen eigenen Motor unabhängig in seiner Extension und Rotation gesteuert. Die Entfernung eines Hypophysen-Tumors erschien so in einem humanen Schädelmodell durchführbar. Morimoto et al. schlagen eine personalisierte Lösung vor, bei der basierend auf der Fragestellung und entsprechender Bildgebung Patienten-spezifische konzentrische Rohre hergestellt werden [124]. Dieser Ansatz wurde allerdings zunächst nur für urologische Patienten ausgeführt.

Die vollautomatische Keilbeinhöhlen-Operation wurde 2005 von der Erlanger Arbeitsgruppe in einem Kadavermodell realisiert [20]. Hierfür wurde ein Bohrsystem auf einen Roboter-Arm mit sechs Freiheitsgraden montiert und mit einem optischen Navigationssystem gekoppelt. Die durchschnittliche Genauigkeit des gesamten Systems lag bei 1,5 mm. Über eine Weiterentwicklung des Systems ist allerdings bisher nicht berichtet worden.

Im Folgenden werden 3 Endoskop-Halter vorgestellt, welche dem Operateur den gleichzeitigen Einsatz von 2 Instrumenten ermöglichen. Der SOLOASSIST Endoskop-Halter (AKTORmed, Regensburg) ist ursprünglich für die Abdominalchirurgie entwickelt worden und wird nun in Kadaverstudien für die Anforderungen der lateralen Nasennebenhöhlen- und Schädelbasischirurgie adaptiert ( $>$ Abb. 5a) [86]. Der Endoskop-Halter kann direkt über einen kleinen Joystick gesteuert werden, welcher an die starren Standard-Instrumente montiert wird. In den publizierten Kadaverstudien konnten die wichtigsten Landmarken an der lateralen und frontalen Schädelbasis sicher identifiziert werden.

Eine Sprachkontrollfunktion mit 23 Einzelbefehlen war in dem alternativen AESOP Endoskop-Halter (Computer Motion, Goleta, CA) integriert, so dass auch hier 2 Instrumente gleichzeitig eingesetzt werden konnten. Zusätzlich konnten anatomische Landmarken gespeichert und später durch ein kurzes Kommando erneut visualisiert werden. Nathan et al. demonstrierten mithilfe des AESOP Systems den Zugang zur Sella turcia in einem humanen Kadavermodell [126]. Für das selbe System wurde kurze Zeit später ein zusätzliches Modul entwickelt, mit dem das Endoskop über die Pupillenbewegung des Operateurs gesteuert werden konnte [4]. Der AESOP Endoskop-Halter war als eigenständiger Baustein in den ZEUS-Roboter integriert, 
a

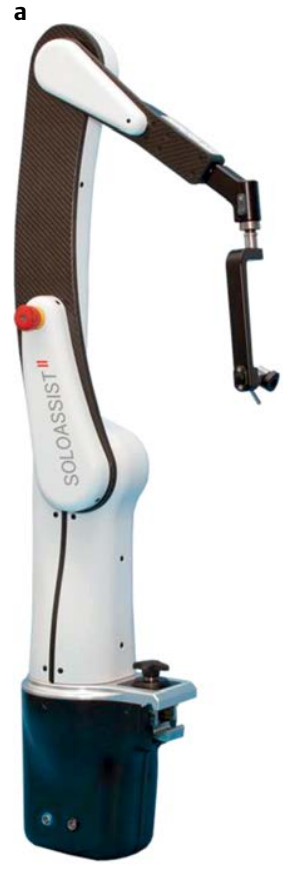

b

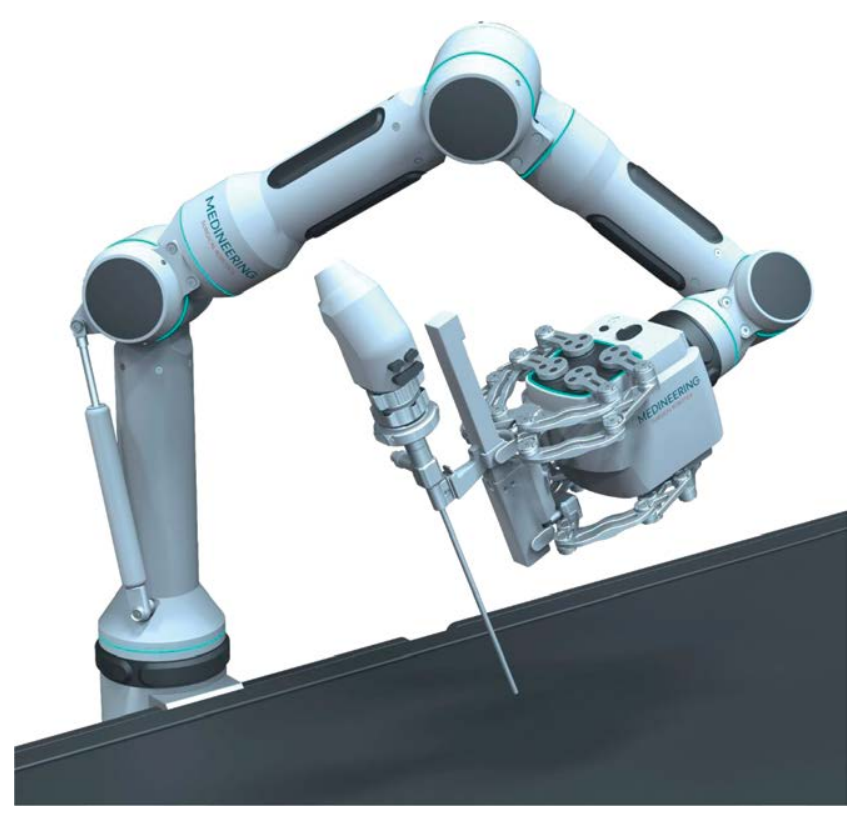

- Abb. 5 a Die neueste Version des SOLOASSIST automatisierten Endoskophalters für minimalinvasive Nasennebenhöhlen-Chirurgie (AKTORmed, Regensburg). b Der robotische Endoskophalter von Medineering (München) ist für die Nasennebenhöhlen-Chirurgie entwickelt worden und steht kurz vor der klinischen Zulassung.

welcher die FDA-Zulassung 2001 erhalten hatte. Das AESOP- und ZEUS-System werden nicht mehr hergestellt und vertrieben. Vergleichsweise jung ist der Endoskop-Halter der Firma Medineering (München), welcher speziell für die Nasennebenhöhlen- und Schädelbasischirurgie entwickelt wird ( $\bullet \mathbf{A b b}$. $\mathbf{5 b}$ ). Das System besteht aus einem mehrgelenkigen Haltearm ohne eigenen Antrieb sowie aus einer kleineren Robotereinheit, welche am Ende des Haltearms befestigt wird. Die Robotereinheit wird über einen Fuß-Joystick bewegt und verfügt über sieben Freiheitsgrade. Erste Kadavertests an den Nasennebenhöhlen und der Schädelbasis sind durch unsere Arbeitsgruppe erfolgreich durchgeführt worden, und die Zulassung für den europäischen Raum wird in Kürze erwartet [51].

Das oben beschriebene $\mathrm{FLEX}^{\circledR}$-System wurde hauptsächlich für den Einsatz im Oropharynx entwickelt. In einem humanen Kadavermodell konnte unsere Arbeitsgruppe das System für die Visualisierung der Nasennebenhöhlen, der vorderen Schädelbasis und des Hirnstamms adaptieren [161]. Allerdings ist die Miniaturisierung des Endoskops für einen transnasalen Zugang bisher noch nicht ausreichend fortgeschritten, und die Resektion der knöchernen Strukturen mit den vorhandenen Instrumenten bleibt eine bisher unüberwindbare Herausforderung.

Für den Einsatz des DaVinci-Systems an der Schädelbasis sind verschiedene Zugänge beschrieben worden, welche je nach Operationsgebiet spezifische Vorteile haben können [144]. Der kombinierte transnasale/transorale Zugang ist zunächst relativ atraumatisch, da keine äußeren Zugangsschnitte angelegt werden müssen ( Abb. 6a). In dem humanen Kadavermodell von Carrau et al. konnte über diesen Zugang die Fossa infratemporalis, die Clivusregion und der kraniozervikale Übergang dargestellt werden. Ebenso konnte die Arbeitsgruppe in 2 klinischen Fällen einen malignen Tumor aus dem Nasopharynx resezieren. Hierbei wurde der kranielle Anteil des Tumors mittels herkömmlichen starren Instrumenten über einen transnasalen Zugang und der kaudale Tumoranteil mittels TORS reseziert [28]. Ebenso wurde der transorale Zugang in einem klinischen Fall getestet, in dem die Resektion des Dens axis bei Kompressionssyndrom der Arteria basilaris erfolgreich durchgeführt wurde [101] ( $\triangleright$ Abb. 6b). Allerdings scheint hier der Einsatzbereich durch die geringe Auswahl der verfügbaren Knocheninstrumente, wie z. B. ein Bohrer, limitiert zu sein. Der kombinierte transnasale/transorale Zugang beschreibt auch einen Aufbau, bei dem die Kamera des DaVinci-Systems transnasal eingeführt wird und für die Arbeitsinstrumente der transorale Zugang gewählt wird. Erstmals beschrieben wurde dieser Zugang in einem humanen Kadavermodell, welcher die hintere Schädelgrube adressierte [136].

Den kombinierten transnasalen/transantralen Zugangsweg zur Schädelbasis konnten Kupferman et al. in einem humanen Kadavermodell demonstrieren. Auch hier erfolgte die Visualisierung der Schädelbasis über die transnasal platzierte Kamera des DaVinci-Systems. Die Arbeitsinstrumente werden über den transantralen Zugang nach midfacial degloving eingeführt. Über diesen Zugang konnten die mediale Orbita, die Siebbeinzellen, die Riechrinne, die Keilbeinhöhle, die Fossa pterygopalatina und der Clivus dargestellt werden [59]. Der leicht modifizierte transantrale/transmaxilläre Zugang ist laut derselben Arbeitsgruppe auch ausreichend zur Defektdeckung an der Schädelbasis im Kadavermodell [89].

Beim transnasalen/transzervikalen Zugang werden die Instru- 


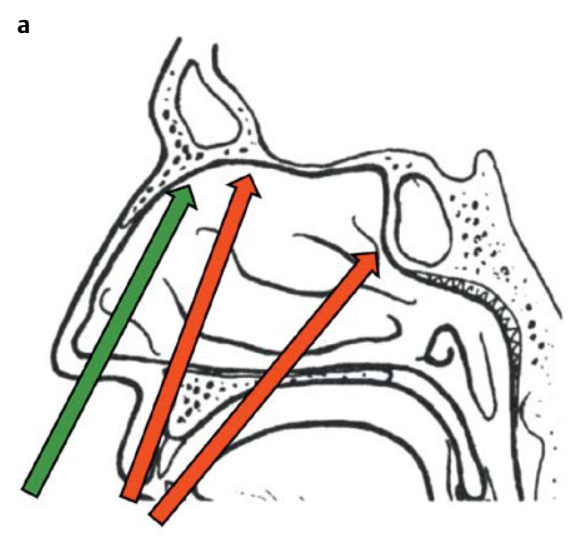

C

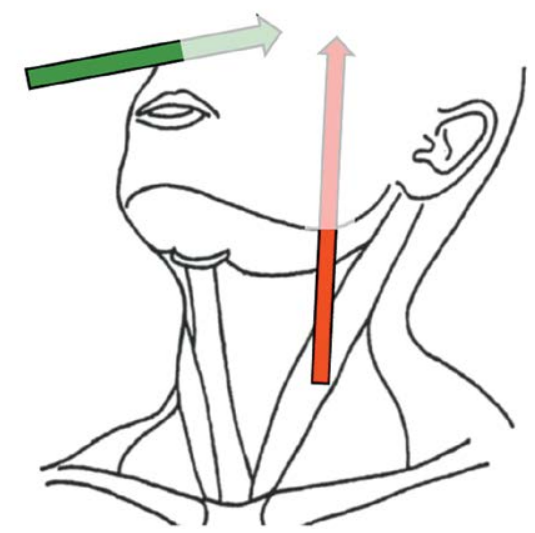

b

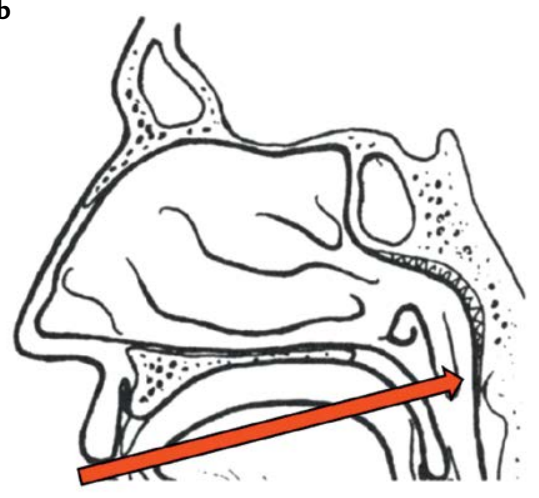

d

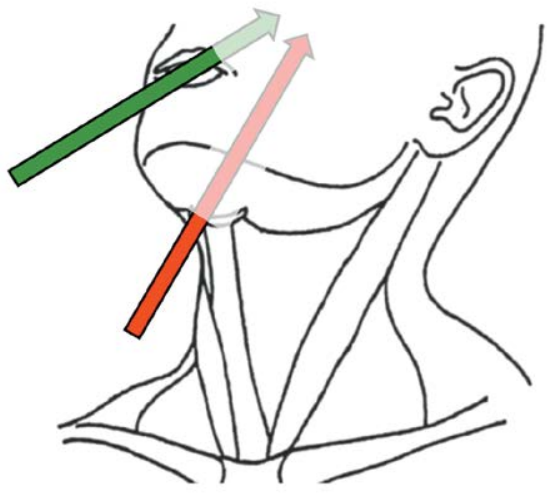

- Abb. 6 Die Roboter-assistierten Zugänge zur Schädelbasis umfassen a transnasal in Kombination mit transoral oder transantral; b rein transoral; c transnasal in Kombination mit transzervikal; und d transoral in Kombination mit suprahyoidal. Copyright: Schuler PJ.

mente über einen Hautschnitt am beidseitigen Kieferwinkel eingeführt ( $>$ Abb. 6c). Der Zugang wird mit stumpfer Präparation erweitert und während des Eingriffs mit einem Trokar offengehalten. Das Kamera-System wird transnasal eingeführt [37]. Dieser Zugang hat zwar eine höhere Invasivität als der transorale Zugang, kann aber bei Patienten mit ungünstigem Zahnstatus oder maxillärer Retrognathie vorteilhaft sein, um den Oberkiefer zu schützen. Zusätzlich stehen die Arbeitsinstrumente so in einem verbesserten Winkel zueinander, was die Manipulation der Schädelbasis erleichtert. Ebenso kann dieser transzervikale Zugang der Instrumente mit einer transoralen Kameraführung kombiniert werden, um die Darstellung der Keilbeinhöhle, des Clivus, der Sella und der Hypophyse in einem humanen Kadavermodell zu erreichen [130].

Abschließend konnte die Arbeitsgruppe um McCool et al. den Zugang zur Fossa infratemporalis über einen kombinierten transoralen/suprahyoidalen Zugang im humanen Kadavermodell demonstrieren [115]. Hierfür wurde ein Instrumentenarm über einen suprahyoidalen Hilfsschnitt zunächst in der Vallecula platziert ( $\triangleright$ Abb. 6d). Das Kamera-System und der zweite Instrumentenarm wurden transoral eingeführt. So konnten die Hirnnerven IX-XII, die Arteria carotis und die Vena jugularis an ihrem Eintritt in die Schädelbasis detailliert dargestellt und manipuliert werden.

Zusammenfassend ist die klinische Anwendung aller oben beschriebenen Systeme für die Nasennebenhöhlen- und Schädelbasis- chirurgie bisher nicht praktikabel [177]. Die maßgeblichen Limitierungen umfassen das Fehlen eines Bohrers und einer Absaugvorrichtung sowie der extensive Durchmesser der Arbeitsinstrumente (>4 mm). Außerdem ist das fehlende haptische Feedback an der Schädelbasis aufgrund der wechselnden Knochen- und Weichteilstrukturen von besonderem Nachteil.

\section{Schilddrüse}

In der Schilddrüsen-Chirurgie ist neben dem offenen Zugangsschnitt auch der transaxilläre, endoskopische Zugang weit verbreitet, der besonders in fokussierten Zentren an großen Patientenkollektiven beschrieben worden ist $(n=581)$ [72]. Mit Einführung des DaVinci ${ }^{\circledR}$ Systems konnte 2005 auch erstmals die Roboter-assistierte Schilddrüsen-Operation über einen transaxillären Zugang bei einem Patienten mit solitärem Schilddrüsen-Knoten durchgeführt werden [107]. Besonders in der Region Südostasien besteht eine große Nachfrage für die Roboter-assistierte Schilddrüsen-Operation, da die offen sichtbare Narbe im Jugulum dort als extrem störend empfunden wird. Dementsprechend können die Operateure aus dieser Region auch gute Ergebnisse in Kohorten mit tausenden von Patienten aufweisen [9, 99]. Aber auch nicht-asiatische Arbeitsgruppen verwenden diesen Zugang in deutlich geringeren Fallzahlen mit überwie- 


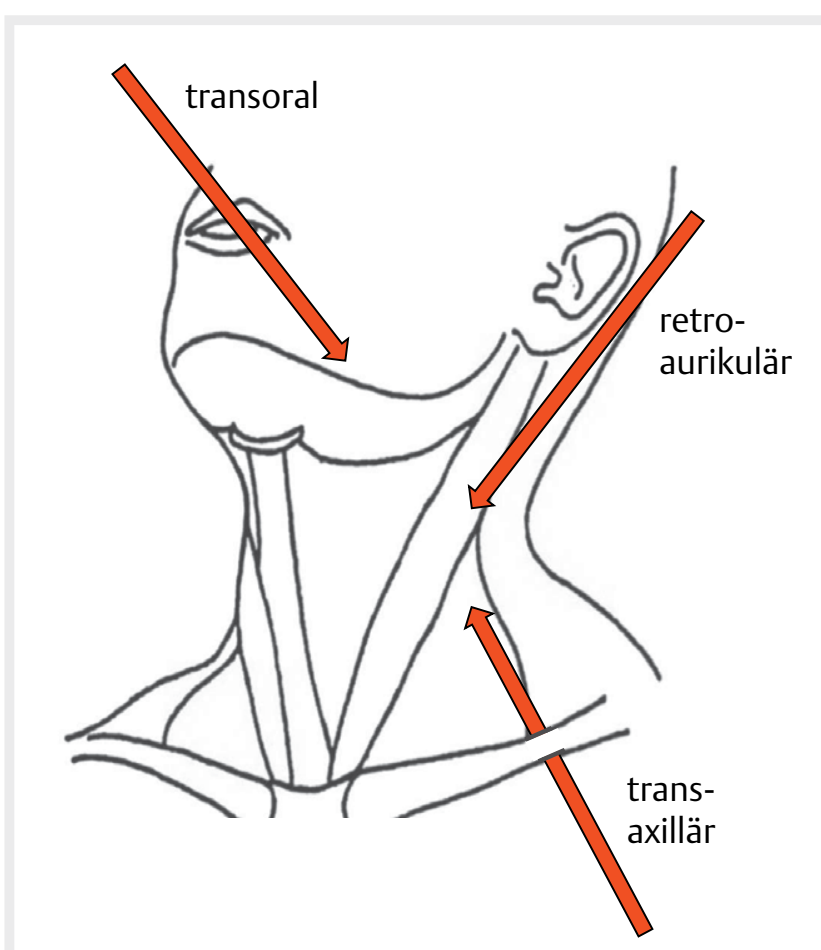

Abb. 7 Die Roboter-assistierte Neck dissection ist über einen transoralen, retroaurikulären oder transaxillären Zugangsweg durchführbar Copyright: Schuler PJ.

gend nicht-malignen Erkrankungen [1, 90, 92].

Mehrere südkoreanische nicht-randomisierte Studien vergleichen die Vor- und Nachteile verschiedener chirurgischer Zugänge in der Schilddrüsenchirurgie. Lee et al. zeigten in einer onkologischen Multicenter-Studie für die Roboter-assistierte Gruppe eine signifikant kürzere Operationszeit bei totaler Thyroidektomie als für die endoskopisch operierte Vergleichsgruppe [100]. Der kombinierte transaxilläre/transpectorale Zugang verspricht allerdings keine operativen Vorteile, sondern nur ein nachteiliges kosmetisches Resultat [165]. Der Langzeitverlauf von postoperativen Stimmbeschwerden wurde von Song et al. in einer nicht-randomisierten Studie untersucht. In diesem Patientenkollektiv erholten sich eventuelle Stimmbeschwerden nach Schilddrüsenchirurgie nach Angaben der Operateure in der Roboter-assistierten Gruppe schneller als nach offener Chirurgie [166]. Die Patienten wurden hierfür über einen Zeitraum von 2 Jahren mittels Fragebogen untersucht.

Die Komplikationen des transaxillären Zugangs sind gut dokumentiert und beinhalten unter anderem persistierende Hypokalziämie $(1,1 \%)$ und dauerhafte Rekurrensparese (0,27\%). Die Roboter-assoziierten Komplikationen waren Hautperforationen im Zugangsbereich $(0,1 \%)$ und Lagerungsschäden der oberen Extremität $(0,13 \%)$ $(n=3,000)$ [9]. Speziell für die transaxilläre Schilddrüsen-Chirurgie wurde von einer japanischen Arbeitsgruppe ein Wundsperrer mit integrierter Kamera entwickelt [68]. Dieser erleichtert die Anlage des subkutanen Zugangstunnels, bevor das DaVinci-System angelegt wird.

Bei Karzinom-Patienten, welche sowohl eine Thyroidektomie als auch eine Neck dissection benötigen, können beide Prozeduren über den transaxillären Zugang durchgeführt werden. Alternativ wird der gesamte Eingriff über einen beidseitigen retroaurikulären Zugang durchgeführt, was laut Terris et al. in einer kürzeren Operationszeit und schnelleren Erholung der Patienten resultiert $(n=15)$ [174]. Die Vorteile des retroaurikulären Zugangs auch für die Schilddrüsen-Chirurgie konnten später durch weitere Untersuchungen bestätigt werden $(n=90)[26,44]$. Prinzipiell ist die totale Thyroidektomie und beidseitige zentrale Neck dissection über einen einseitigen retroaurikulären Zugangsschnitt durchführbar, was dann aber mit einer deutlich längeren Operationszeit verbunden ist [25].

Der transorale Zugangsweg zur Schilddrüse wurde von amerikanischen und südkoreanischen Arbeitsgruppen mit dem Ziel entwickelt, vollständig auf sichtbare Hautnarben verzichten zu können [149]. Die endoskopische sublinguale Thyroidektomie wurde erstmals durch Benhidjeb 2009 beschrieben [11] und in Kadaverstudien auch für die Roboter-assistierte Chirurgie adaptiert [150]. Im Rahmen der technischen Weiterentwicklung wurde der enorale Zugang vom sublingualen Raum in das Vestibulum verlegt und hierdurch die Interferenz der Instrumente mit den Kieferknochen deutlich reduziert [150]. Bei diesem Zugang muss allerdings die transiente Alteration des Nervus mentalis berücksichtigt werden, welche sich im Normalfall nach wenigen Wochen zurückbildet $[98,153]$.

Eine angeregte Diskussion wurde durch eine Publikation von Lee et al. entfacht, welche Patienten nach Roboter-assistierter Schilddrüsen-Chirurgie ein verbessertes Körperbewusstsein zuschreibt [102]. Auf der einen Seite muss berücksichtigt werden, dass die Studie in einer jungen, nicht-randomisierten asiatischen Patientengruppe durchgeführt wurde und die Beobachtungen nur bedingt auf andere Kulturkreise übertragbar sind [105]. Auf der anderen Seite ist besonders bei Karzinom-Patienten abzuwägen, welchen Stellenwert die kosmetische Erscheinung gegenüber der onkologischen Sicherheit während des Eingriffs einnehmen darf. Hierzu konnte die qualitativ hochwertige und detaillierte Meta-Analyse von Chai et al. in Bezug auf die onkologische Sicherheit keinen Nachteil für die Roboter-assistierte Chirurgie identifizieren, so dass der Eingriff zumindest für erfahrene Operateure mit hohen Fallzahlen als sicher angesehen werden kann [30].

\section{Halsweichteile}

Ebenso wie bei der Schilddrüsen-Chirurgie wird auch die Entwicklung der Roboter-assistierten Neck dissection (RAND) stark von den südkoreanischen Arbeitsgruppen dominiert. Besonders an der Yonsei Universität in Seoul werden RAND-Eingriffe sowohl von den Kollegen der HNO als auch der Chirurgie durchgeführt. Auch hier sind hauptsächlich kosmetische Bedürfnisse die treibende Kraft, welche sich in unseren Kulturkreisen nicht widerspiegeln. Aktuell werden 4 verschiedene Zugänge zur RAND beschrieben ( transaxillär, (II) retroaurikulär, (III) der kombinierte transaxilläre und retroaurikuläre TARA-Zugang, sowie (IV) transoral.

Der transaxilläre Zugang wurde erstmals durch Kang et al. 2010 in 33 Patienten mit Schilddrüsenkarzinom und zervikaler Metastasierung beschrieben [73]. Die unilaterale modifiziert-radikale Neck dissection wurde gemeinsam mit der beidseitigen Thyroidektomie durchgeführt. Das operative Vorgehen ist in der oben genannten Publikation detailliert beschrieben. Die durchschnittliche Anzahl der entfernten Lymphknoten war 6,1 $\pm 4,4$ im medialen und 27,7 $\pm 11,0$ im lateralen Kompartment. Der transaxilläre Zugangsweg bietet sich bei Schilddrüsenkarzinomen an, ist aber bei Kopf-Hals-Karzinomen 
- Tab. 1 Aktuelle klinische Studien zur Roboter-assistierten Chirurgie im Kopf-Hals-Bereich (www.clinicaltrials.gov).

\begin{tabular}{|c|c|c|c|c|c|c|}
\hline & Studien-\# & Land & Zeitraum & Status & Inhalt & Patientenzahl \\
\hline \multicolumn{7}{|c|}{ Fallserien } \\
\hline S1 & NCT00473564 & USA & $2007-2015$ & geschlossen & TORS, Fallserie, DaVinci & 36 \\
\hline S2 & NCT02262247 & USA & 2014-2015 & geschlossen & TORS, FLEX ${ }^{\circledR}$-System & 80 \\
\hline S3 & NCT01059357 & USA & 2010-2014 & geschlossen & TORS, Fallserie, DaVinci & 60 \\
\hline S4 & NCT01819480 & USA & 2013-2019 & offen & TORS, Fallserie, DaVinci & 85 \\
\hline S5 & NCT02225496 & USA & 2014-2019 & offen & TORS, Fallserie, DaVinci & 140 \\
\hline S6 & NCT01473784 & USA & $2007-2020$ & offen & TORS, Fallserie, DaVinci & 360 \\
\hline S7 & NCT02159703 & USA & 2014-2017 & offen & Radiatio nach TORS (HPV+) & 60 \\
\hline S8 & NCT02072148 & USA & 2014-2019 & offen & Radiatio nach TORS (HPV+) & 200 \\
\hline S9 & NCT03107182 & USA & $2017-2020$ & offen & Induktion vor TORS oder RCT & 56 \\
\hline S10 & NCT02760667 & USA & $2015-2020$ & offen & Induktion vor TORS oder TLM & 20 \\
\hline S11 & NCT01187160 & USA & $2009-2014$ & geschlossen & Schlafapnoe-Syndrom & 75 \\
\hline S12 & NCT01107795 & USA & 2010-2019 & offen & Schlafapnoe-Syndrom & 75 \\
\hline S13 & NCT02269020 & Frankreich & 2015-2018 & offen & Neck dissection & 3 \\
\hline S14 & NCT02274493 & USA & 2015-2019 & offen & Latissimus Transplantat & 15 \\
\hline S15 & NCT02743442 & Frankreich & 2016-2017 & offen & Hypophysen-OP & 8 \\
\hline S16 & NCT02792322 & USA & 2016-2018 & offen & TORS in sitzender Position & 50 \\
\hline S17 & NCT02517125 & Frankreich & $2015-2025$ & offen & DaVinci System (Xi) & 150 \\
\hline S18 & NCT03010813 & Hong Kong & 2016-2017 & offen & DaVinci System (SP) & 60 \\
\hline S19 & NCT01488669 & Südkorea & 2011-2013 & geschlossen & Neck dissection & 26 \\
\hline \multicolumn{7}{|c|}{ Randomisierte Studien } \\
\hline S20 & NCT01898494 & USA & $2013-2023$ & offen & TORS und Radiatio & 377 \\
\hline S21 & NCT02002182 & USA & 2013-2019 & offen & Vakzinierung vor TORS & 30 \\
\hline S22 & NCT02292914 & Brasilien & 2014-2017 & offen & TORS vs. Standard Chirurgie & 1120 \\
\hline S23 & NCT02984410 & Europa & $2017-2020$ & offen & Radiatio vs. Chirurgie (mit TORS) & 170 \\
\hline S24 & NCT01590355 & Kanada & $2012-2021$ & offen & Radiatio vs. TORS & 68 \\
\hline
\end{tabular}

aufgrund des langen Tunnels zu den kraniellen Lymphknotenstationen nicht praktikabel. Der alternative retroaurikuläre Zugang über einen modifizierten Facelift-Schnitt konnte erstmals durch Blanco et al. 2012 in einer Kadaverstudie beschrieben werden [14]. AnschlieBend wurden im Rahmen einer prospektiven, nicht-randomisierten klinischen Studie in Südkorea 26 Patienten mit Oropharynx-Karzinom ohne zervikale Metastasierung entweder der retroaurikulären RAND oder der konventionellen, offenen Chirurgie zugeteilt (NCT01488669, > Tab. 1). Interessanterweise waren die Komplikationsraten und die Anzahl der entfernten Lymphknoten in beiden Gruppen gleich [97]. Sehr ähnliche Studien, welche diese Ergebnisse bestätigen, sind später für Patienten mit Kopf-Hals-Karzinom mit und ohne zervikale Lymphknoten-Metastasen $\left(\mathrm{cNO} / \mathrm{CN}^{+}\right)$publiziert worden $[78,106,172]$. Wenig überraschend war die signifikant verlängerte Operationszeit für die RAND-Gruppe in allen Studien. Allerdings wird die Operationszeit bei RAND-Eingriffen durch entsprechendes Training deutlich reduziert. In der Publikation von Kim et al. wurden 90 Kopf-Hals-Karzinom-Patienten durch einen einzigen Operateur mittels RAND operiert [77]. Sowohl in der Gruppe der modifiziert radikalen Neck dissection als auch bei der supraomohyoidalen Neck dissection konnte die Operationszeit im Verlauf der Studie si- gnifikant reduziert werden (298 ->212; 226 -> 106 Min).

Durch die positiven Erfahrungen der Operateure mit der RANDTechnik konnte die Indikation auch auf nicht-onkologische Patienten erweitert werden. So zeigten Lee et al. in einer nicht-randomisierten, prospektiven Studie die Möglichkeiten der Roboter-assistierten $(n=13)$ und der endoskopischen Submandibulektomie $(n=22)$ auf [96]. Beide Verfahren erfolgten mit retroaurikulärem Zugangsschnitt und dauerten mit 63 bzw. 65 Min in etwa gleich lang. Kein Eingriff musste in einen offenen Zugang konvertiert werden und die kosmetischen Ergebnisse zeigten keinen signifikanten Unterschied.

Wie oben beschrieben ist der retroaurikuläre Zugang dem transaxillären Zugang bei der Neck dissection in den kranialen Lymphknoten-Kompartimenten überlegen. Ist eine ausgedehnte Neck dissection in den kranialen und kaudalen Kompartimenten geplant, so kann, wie von Kim et al. beschrieben, die Kombination aus transaxillärem und retroaurikulärem Zugang sinnvoll sein $(n=7)$ [80]. Der kombinierte Zugang wird sowohl bei Patienten mit Kopf-Hals-Karzinom als auch bei Schilddrüsen-Karzinomen durchgeführt und dauert länger als der konventionelle, offene Zugang, produziert dafür aber vorteilhafte kosmetische Ergebnisse $(n=22)$ [79]. Die weitere Entwicklung zeigt, dass auch die beidseitige Thyroidektomie und 

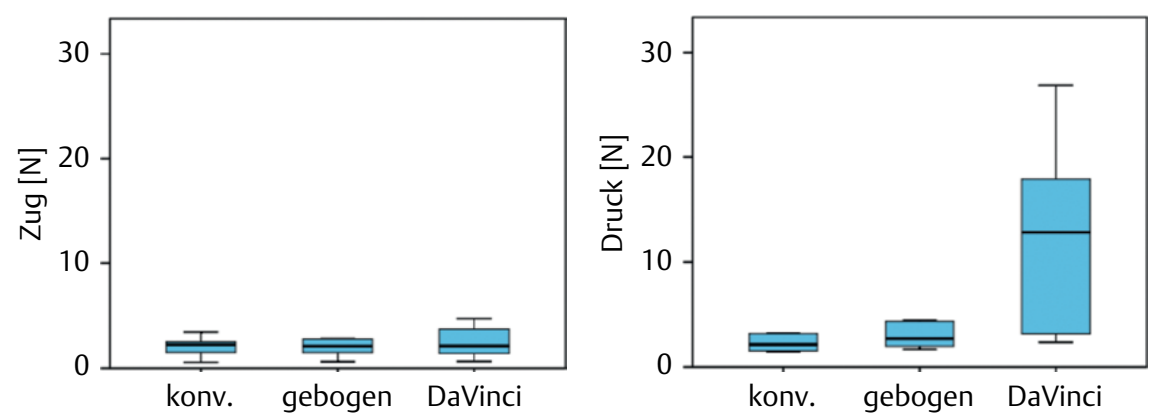

- Abb. 8 In experimentellen Studien ist die intraoperative Druckentwicklung mit dem Roboter-System signifikant höher als mit konventionellen Instrumenten. Copyright: Schuler PJ.

modifiziert-radikale Neck dissection über einen einzigen ipsilateralen retroaurikulären Zugangsschnitt Roboter-assistiert durchgeführt werden kann $(n=4)$ [25]. Da die Operationsdauer regelmäßig bei über 5 Stunden lag, muss allerdings die Sinnhaftigkeit und besonders die onkologische Sicherheit der Prozedur in Frage gestellt werden. Im europäischen Raum wird das kosmetische Ergebnis besonders nach onkologisch motivierter Operation nicht ähnlich hoch bewertet, wie in asiatischen Kulturkreisen. Viel mehr werden in unserem Raum die bestmöglichen onkologischen Resektionsgrenzen gegenüber kosmetischen Bedenken bevorzugt. Daher werden die Roboter-assistierte Schilddrüsen-Operation und Neck dissection nicht regelmäßig angeboten.

Abschließend wurde durch verschiedene Arbeitsgruppen die Bedeutung der retropharyngealen Lymphknotengruppe bei Patienten mit Kopf-Hals-Karzinom hervorgehoben. Byeon et al. zeigten 2013, dass die transorale Neck dissection im retropharyngealen Kompartiment sicher und effektiv durchgeführt werden kann $(n=5)$ [24]. Die Lymphknoten-Exstirpation erfolgte jeweils in der Tiefe des Wundbetts nach Resektion eines Tonsillen- oder Hypopharynx-Karzinoms. Obwohl jeweils nur einer oder 2 Lymphknoten reseziert werden konnten, zeigten 4/5 Patienten einen Tumorbefall dieser Lymphknoten. Später wurden diese Ergebnisse durch eine amerikanische Arbeitsgruppe bestätigt $(n=30)$ [178]. Weiterhin unklar ist der ideale zeitliche Abstand zwischen Resektion des Primärtumors und indizierter Neck dissection. Bei Tumoroperationen mittels TORS ist für die Neck dissection eine aufwendige Umlagerung des Patienten und oft auch ein Wechsel des Operationsraums notwendig, was in vielen Fällen bisher nicht praktikabel ist. Frenkel et al. zeigten nun für den Raum New York im Zeitraum 2008-2012, dass die Neck dissection in $76 \%$ der Fälle zeitgleich mit der TORS-basierten Tumorresektion durchgeführt wurde. Bei allen anderen Patienten erfolgte die Neck dissection entweder im Vorfeld (4\%) oder 2-zeitig nach der Tumoroperation (20\%).

\section{Haptik}

Die aktuellen Robotersysteme haben den Nachteil, dass sie keine oder nur sehr begrenzt haptische Information an den Operateur weiterleiten können [182]. Die fehlende Haptik kann aber oft durch die visuelle Kompensation sehr gut ausgeglichen werden, was zu einer niedrigen Akzeptanz der haptischen Feedback-Systeme führt [117].
Besonders wenn das Roboter-System eine 3D-Bildgebung besitzt, ist die erhöhte Kraftaufwendung im Allgemeinen durch die Deformation des Gewebes erkennbar. Ebenso wird die Steifigkeit durch eine ausbleibende Gewebedeformation angezeigt. Auf der anderen Seite kann die fehlende Haptik z. B. dazu führen, dass Knoten während eines Eingriffs zu locker sitzen oder Fäden auch von erfahrenen Operateuren abgerissen werden [12]. Auch wird vermutet, dass die fehlende Haptik bei endovaskulären Roboter-Eingriffen zu einem erhöhten Risiko einer Gefäßverletzung führt [6]. Bei der Auswertung von laparoskopischen Eingriffen, welche durch angehende Chirurgen durchgeführt worden waren, konnte gezeigt werden, dass über die Hälfte aller Fehler durch überschießende Kraftanwendung verursacht wurde [173]. Leider fehlen entsprechende Untersuchungen im HNO-Bereich. Trotzdem könnten diese Fehler durch entsprechendes haptisches Feedback zumindest teilweise verhindert werden. Unsere Arbeitsgruppe konnte in einem ex vivo Experiment zeigen, dass auch mit dem DaVinci-System der Druck und das Drehmoment auf das Gewebe im Operationsbereich mehrfach erhöht ist im Vergleich zu konventionellen starren Instrumenten und auch zu gebogenen Instrumenten ( $>$ Abb. 8). Die Probanden des Experiments mussten mit verschiedenen Instrumenten Teile eines Silikonkissens unter kontinuierlicher Kraftmessung resezieren. Diese verstärkte Krafteinwirkung auf das Gewebe kann eine erhöhte Verletzungsgefahr mit erhöhter Morbidität des Patienten bewirken.

Zunächst besteht die Frage, wie das Roboter-System die Charaktereigenschaften des Gewebes aufnehmen kann ( $>$ Abb. 9). Im einfachsten Fall geschieht dies über kapazitive Drucksensoren, in denen sich bei Druckeinwirkung die elektrische Kapazität verändert und ein elektrisches Signal weitergeleitet wird. Auf ähnliche Weise wird die Verbiegung von Instrumenten bei seitlichem Druck gemessen und signalisiert [154]. Für das DaVinci-System konnten diese Überlegungen schon relativ weit umgesetzt werden. Kim et al. haben Drucksensoren in die Innenseite einer DaVinci-Pinzette integriert, über welche ex vivo die Druckeinwirkung in 4 Freiheitsgraden gemessen werden kann [76]. In einem in vivo Tiermodell konnten Wottawa et al. zeigen, dass schon die Übermittlung des eindimensionalen Gewebedrucks an den Operateur die Gewebeschädigung signifikant reduziert [195]. Moderne Systeme kombinieren mehrere Sensoren in einem Instrument, wie z. B. kapazitive Folien und Flüssigkeiten, sowie Temperatursonden, um den Informationsgehalt zu erhöhen [138]. Prinzipiell können die Gewebeeigenschaften auch über den 


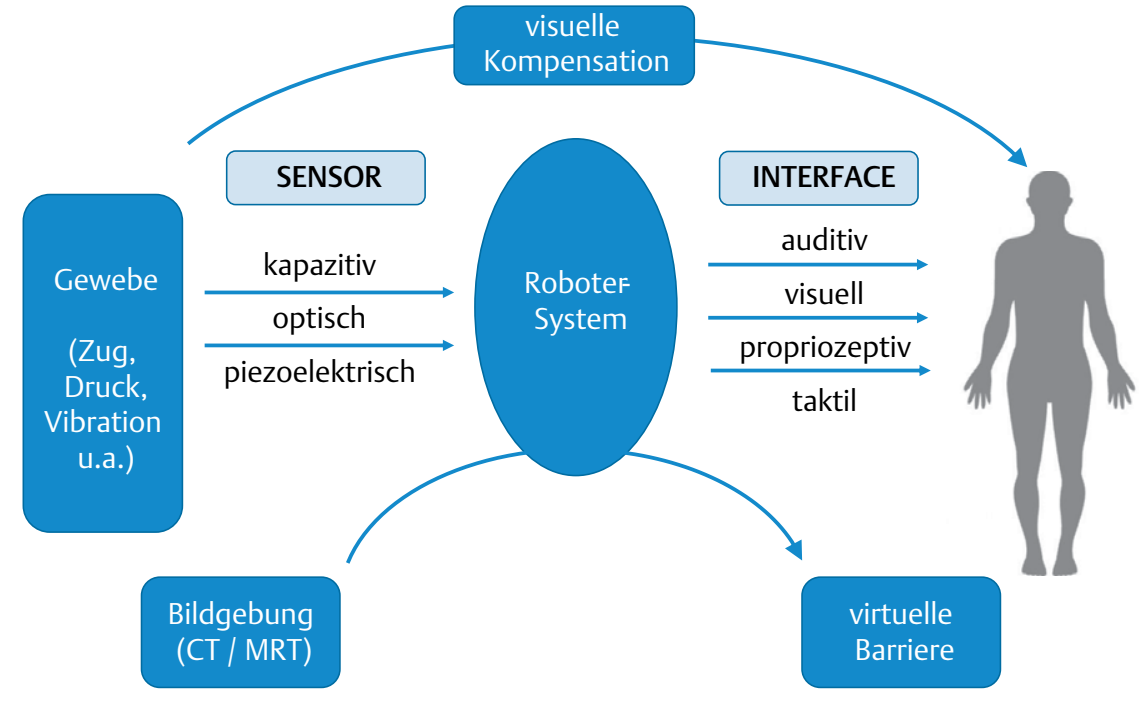

- Abb. 9 Das Roboter-System hat eine sensorische Komponente mit der Informationen über das Gewebe gewonnen werden. Über das haptische Interface interagiert das System mit dem Operateur. Die visuelle Kompensation kann Defizite des Roboter-Systems teilweise ausgleichen. Eine virtuelle Barriere kann mithilfe einer CT/MRT-Bildgebung festgelegt werden. Copyright: Schuler PJ.

piezoelektrischen Effekt oder optoelektronische Sensoren gemessen werden $[69,128]$. Allerdings befinden sich diese Technologien noch in der Grundlagenentwicklung ohne klinische Anwendung.

Auf der anderen Seite müssen die gemessenen Signale an den Operateur weitergeleitet werden. Bei genauerer Betrachtung können hierbei 2 verschiedene Entitäten unterschieden werden, wie der menschliche Körper haptische Signale empfangen kann. Zum einen werden propriozeptive Signale wahrgenommen, welche hauptsächlich über Rezeptoren in den Muskeln und Sehnen aufgenommen werden und Information über die Lage, Kraft und Bewegung des Körpers beinhalten. Auf der anderen Seite werden taktile Signale über die Hautrezeptoren aufgenommen, welche Informationen über Druck, Vibration, Wärme und Schmerz verarbeiten [132]. Bei den konventionellen nicht-robotischen Operationstechniken schöpft der Operateur mithilfe seiner Finger beide Informationskanäle vollständig aus, indem er das Gewebe ausgiebig palpiert. Auch die neuen Roboter-Systeme können in der Theorie taktile Signale an den Operateur weiterleiten. Hierzu sind allerdings komplizierte Apparaturen, wie elektromagnetische Nadeln, temporär verformbare Gewebe, piezoelektrische Kristalle, pneumatische Pumpen oder Wärmesysteme notwendig [10].

Verschiedene präklinische Aufbauten zeigen, dass das taktile Feedback die applizierten Kräfte während einer Prozedur deutlich reduzieren kann. Hierbei ist es unerheblich, ob die Signale mittels pneumatischem Ballon-Interface oder mittels Vibrationsmotor übermittelt werden [104, 138]. Aber die Umsetzung ist aufwendig, und die interindividuellen Unterschiede in der Wahrnehmung sind sehr unterschiedlich ausgeprägt. Zusätzlich gelten die strengen hygienischen Anforderungen bei der Reinigung der Instrumente auch für die taktilen Apparate, was den regelmäßigen Einsatz nochmals erschwert. Daher beschränken sich aktuelle Bemühungen hauptsächlich auf den propriozeptiven Informationskanal, wobei je nach Stärke des Signals die Bewegungen des Operateurs am Manipulator mit- hilfe von elektrischen Motoren gebremst oder eingeschränkt werden [132]. Diaz et al. prüften den Ansatz, ob ein haptisches Signal über ein zusätzliches Fußpedal an den Operateur gesendet werden kann [42]. Allerdings hat sich auch diese Methode bislang nicht durchsetzen können.

Neben den propriozeptiven und taktilen Signalen können Roboter-Systeme Informationen auch über auditive und visuelle Sinneskanäle an den Operateur übertragen ( $\triangleright$ Abb. 9). Die Arbeitsgruppe von Okamura et al. rüstete ein DaVinci-System mit einer zusätzlichen Ton- und Bildanzeige aus, welche dem Operateur die applizierten Kräfte übermittelte. So konnten sie zeigen, dass die Fäden beim Knoten mit visuellem Feedback seltener rissen und die Konsistenz der Kräfte mit dem DaVinci-System sogar besser als mit der Hand war [81, 146]. Ebenso konnten Ly et al. die Vorteile des akustischen Feedbacks darstellen, wenn es mit einer sensorischen Pinzette gekoppelt wurde [110].

Eine alternative Feedback-Methode sind virtuelle Barrieren, welche anhand von präoperativ erstellten Bildgebungen festgelegt werden. Die Barrieren markieren wichtige anatomische Landmarken, wie z. B. große Blutgefäße, und der Operateur kann seine Instrumente nicht über diese Grenzen hinwegbewegen [19]. Ähnlich wie bei den haptischen Feedback-Mechanismen werden die Instrumente an den virtuellen Barrieren mithilfe der elektronischen Motoren des Roboter-Systems blockiert.

Zusammenfassend ist der Vorteil der haptischen Feedback-Systeme zum heutigen Zeitpunkt vielfach nachgewiesen worden. Der regelmäßige Einsatz wird aber erschwert durch die technischen, finanziellen und hygienischen Herausforderungen [46].

\section{Kosten}

Aufgrund des materiellen Mehraufwandes ist der finanzielle Mehraufwand bei roboterunterstützten Operationen erheblich. Da das DaVinci-System lange das einzige zugelassene System war, liegen 
bisher nur hier detaillierte Kostenanalysen vor. Nach Berechnungen von Dombree et al. liegen die Kosten für eine totale Laryngektomie in Belgien um ca. $90 \%$ höher (3,581 vs. 6,767 Euro), wenn eine Roboter-assistierte Operation durchgeführt wird [43]. Während bei den Roboter-Operationen die Kosten für das System und die spezifischen Instrumente am meisten ins Gewicht fallen, sind dies bei den konventionellen Eingriffen die Personalkosten. Eine Kostenäquivalenz kann laut den Autoren auch dann nicht erreicht werden, wenn für die Berechnung die Anzahl der operierten Patienten maximiert und die Operationsdauer minimiert wird. Unterstützt wird diese Ungleichheit der Kosten dadurch, dass die Hersteller der Robotersysteme grundsätzlich ein Monopol für die Operationsinstrumente haben, welche jeweils nur mit dem eigenen System verwendet werden können [64]. Auch im japanischen Krankenkassensystem werden die zusätzlichen Ausgaben der Roboter-Chirurgie kritisch gesehen. So muss ein Zentrum jährlich mindestens 300 Roboter-assistierte Mediastinoskopien durchführen, damit die Kostenäquivalenz gegenüber den Video-assistierten Eingriffen erreicht werden kann [71]. Ebenso konnte in Deutschland für die Roboter-assistierte Prostatektomie aufgrund der hohen Materialkosten keine Kosteneffektiviät nachgewiesen werden [17]. In den USA scheinen die Verhältnisse weniger deutlich zu sein. So konnte laut Byrd et al. für die Roboterassistierte Panendoskopie bei ,Cancer of unknown Primary“ (CUP) die Kosteneffektivität erreicht werden, da sich hierdurch eine höhere Wahrscheinlichkeit ergibt, den Primarius zu finden [27]. In den 206 retrospektiv untersuchten Patienten waren die anfallenden Folgekosten entsprechend geringer. Für Patienten mit obstruktivem Schlafapnoe-Syndrom ( $n=104)$ konnten Golbin et al. im Vergleich zu konventionell operierten Patienten aber eine deutliche Zunahme der Kosten um durchschnittlich 14708 USD feststellen, wenn die Patienten mit TORS operiert wurden [58].

Eine weitere Möglichkeit, um die Kosteneffektivität von RoboterSystemen zu überprüfen, ist die Anwendung von Markov-Modellen, welche versuchen über stochastische Prozesse das Eintreffen von bestimmten Ereignissen vorherzusagen. Auch hier besteht Uneinigkeit über die Kosteneffektivität von Roboter-Systemen. So beschreiben Almeida et al. eine durchschnittliche Kosteneinsparung von 1366 USD und eine Zunahme der qualitätsadaptierten Lebensjahre (QALY) um 0,25 für Oropharynx-Karzinome im Vergleich zu primärer Radiochemotherapie [39]. Die Differenz zugunsten von TORS kann laut den Autoren weiter verbessert werden, wenn die Rate der adjuvanten Radiatio in der TORS Gruppe durch sinnvolle Patientenselektion minimiert wird. Eine ähnliche Studie von Rodin et al. zeigte eine vergleichbare Zunahme der QALY um 0,63 für Patienten mit Oropharynx-Karzinome in Kanada [151]. Allerdings war die primäre Radiochemotherapie mit 123000 USD deutlich günstiger als die TORSTherapie mit 178000 USD, welche eine eventuelle adjuvante Therapie miteinschließt. Zwei weitere Publikationen mit ähnlichem Studienaufbau für Oropharynx-Karzinome sehen die Roboter-Therapie eher im Nachteil. So zeigten Rudmik et al. eine Erhöhung der Kosten um 4959 USD im Vergleich zur primären Radiochemotherapie ohne Zunahme der QALY [152]. Noch deutlicher fiel der finanzielle Nachteil für TORS-Eingriffe in den Berechnungen von Sher et al. aus, welche die Mehrbelastung mit 12100 USD bezifferten ohne eine Veränderung der QALY zu sehen [162]. Alle Markov-Model Studien stammen aus dem nordamerikanischen Raum, sodass im Wesentlichen die gleichen Berechnungsgrundlagen vorlagen.

Ein großer Kostenvorteil für TORS-Patienten wurde in einer retrospektiven Studie von Richmon et al. mit 9601 Patienten errechnet [148]. Der Kostenvorteil entstand hierbei nicht durch den Eingriff selbst, sondern durch die verkürzte Krankenhausliegezeit und die verringerte Anzahl von Magensonden und Tracheostomien in der TORS-Gruppe. Da es sich um eine nicht-randomisierte Studie handelte, wurden gesündere Patienten möglicherweise mit einer höheren Wahrscheinlichkeit der TORS-Gruppe zugeteilt, was die Ergebnisse verfälschen kann. In einer zweiten, ähnlichen Studie von Chung
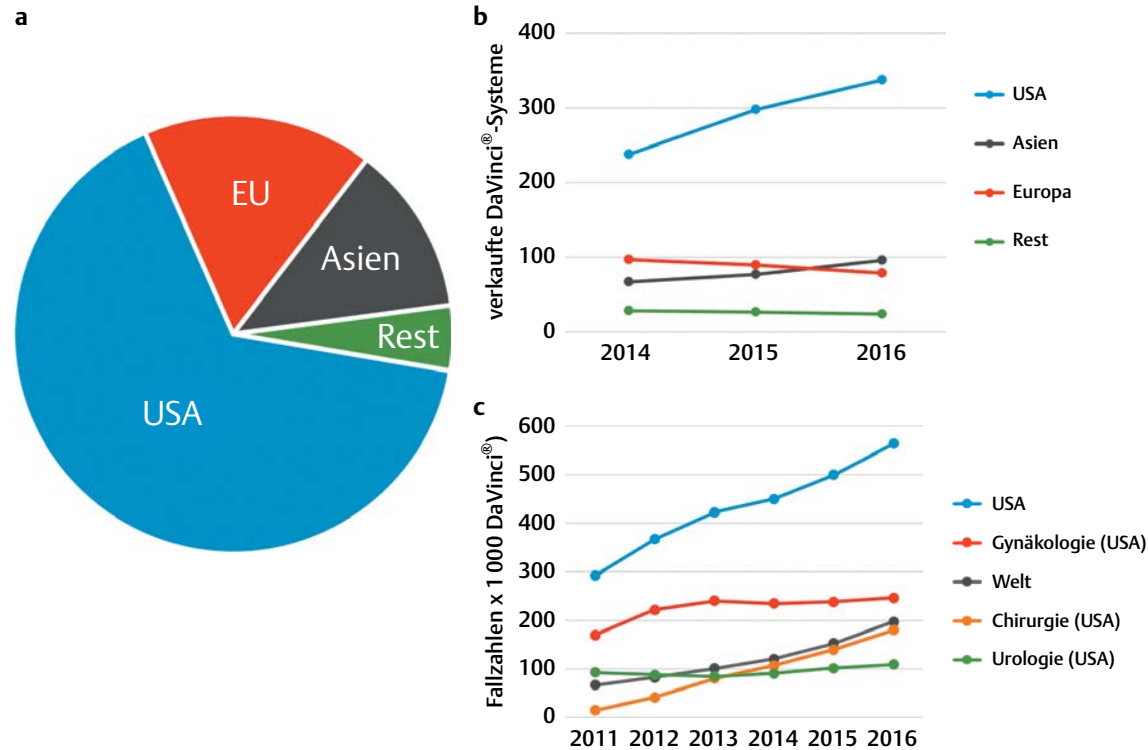

- Abb. 10 a Die meisten DaVinci-Systeme sind in den USA installiert. b Ebenso steigt die Zahl der jährlich verkauften DaVinci-Systeme in den USA weiterhin an. c Korrelierend dazu nimmt besonders in den USA die Anzahl der Roboter-assistierten operativen Eingriffe weiter zu. Copyright: Schuler PJ . 

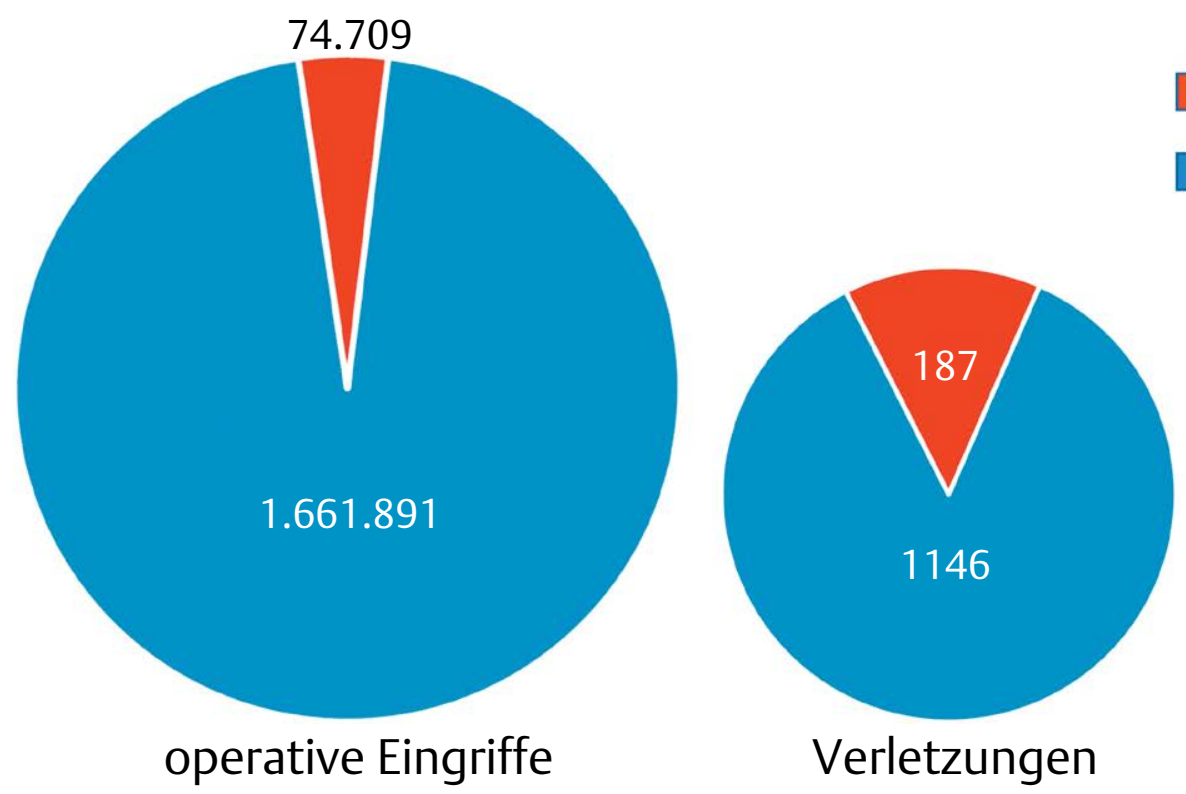

Herz-Thorax, Kopf-Hals

Gynäkologie, Urologie, Allgemein-Chirurgie

(bis 2013)

Abb. 11 Nur ein kleiner Anteil aller Roboter-assistierten Eingriffe wird in der Kopf-Hals- Chirurgie und Herz-Thorax-Chirurgie durchgeführt. Das relative Risiko für Verletzungen oder Todesfälle ist in diesen Disziplinen allerdings erhöht. Copyright: Schuler PJ.

et al. mit 2067 Patienten wurde dieses Problem bei der Auswertung der Daten berücksichtigt. Trotzdem zeigte die Gruppe, welche mit offener Chirurgie behandelt wurde, eine längere Liegezeit, höhere Kosten, sowie eine höhere Anzahl von Magensonden und Tracheostomien als die TORS-Gruppe [34]. Die TORS-Gruppe zeigte hingegen ein höheres Risiko für Dysphagie. Zusätzlich sollte berücksichtigt werden, dass die Kosten des Eingriffs auch von der Wahl der Instrumente durch den Operateur beeinflusst werden. So sind z. B. flexible Laserfasern besonders kostenintensiv und können nicht wiederaufbereitet werden [64]. Laut eigener Berechnungen, entstehen bei einem Eingriff mit dem DaVinci-System zusätzliche Kosten von über 6000 Euro. Anschaffungs- und Wartungsgebühren sind in diesen Kosten anteilsmäßig berücksichtigt und werden in Deutschland bisher nicht über ein spezielles Entgelt für , neue Untersuchungs- und Behandlungsmethoden“ (NUB) rückerstattet [113]. Die aktuellen Anschaffungskosten für das DaVinci-System liegen bei ca. 2 Millionen USD (Xi Model) und ca. 150000 USD jährliche Wartungsgebühr. Die Anschaffung des Flex-Systems kostet aktuell ca. 800000 USD. Je nach Anzahl der jährlich durchgeführten Roboter-gestützten Operationen lässt sich der Preis pro Eingriff entsprechend senken.

\section{Fallzahlen}

Um die Bedeutung von TORS in das gesamte Umfeld der RoboterChirurgie besser einordnen zu können, erscheint die Betrachtung der Systemverteilung und der fächerübergreifenden Fallzahlen sinnvoll. Zuverlässige Zahlen können den Jahresberichten der Firma Intuitive Surgical entnommen werden [67]. Letztes Jahr waren weltweit 3803 DaVinci-Systeme installiert. Neben den 2501 Systemen in den USA waren weitere 644 Systeme in Europa und 476 Systeme in Asien installiert. 182 weitere Systeme entfallen auf andere Kontinen- te wie Afrika, Südamerika und Australien ( $\triangleright \mathbf{A b b} \mathbf{b}$ 10a). Ebenso sind die aktuellen Verkaufszahlen stark vom amerikanischen Krankenhausmarkt dominiert. So werden regelmäßig über die Hälfte aller verkauften Systeme in die USA geliefert ( $\downarrow$ Abb. 10b). Diese ungleiche Verteilung der Systeme spiegelt sich auch in der Anzahl der durchgeführten Prozeduren wider. $>$ Abb. 10c zeigt die Entwicklung der Fallzahlen für den Zeitraum ab 2011. Für den amerikanischen Markt zeigt sich in diesem Zeitraum nahezu eine Verdopplung der Roboter-assistierten Eingriffe. Interessanterweise scheint aber für die Fachgebiete Gynäkologie und Urologie seit mehreren Jahren ein stabiles Plateau erreicht zu sein. Das amerikanische Wachstum basiert somit hauptsächlich auf der anhaltenden Zunahme der Fallzahlen in der Allgemeinchirurgie, welche mittlerweile die urologischen Eingriffe deutlich übertreffen. Auch ist das Verhältnis des amerikanischen Marktes zum übrigen Weltmarkt sehr aufschlussreich. Zwar zeigt sich in den anderen Ländern auch ein stetiges Wachstum der Fallzahlen. Diese liegen aber insgesamt bei nur etwa einem Drittel der amerikanischen Eingriffe. Die Fallzahlen für die Kopf-Hals-Chirurgie sind selbst in den USA so gering, dass dieses Fachgebiet in den Jahresberichten von Intuitive Surgical keine Beachtung finden. Unter Betrachtung dieser Zahlen scheint es verständlich, dass die Weiterentwicklung des DaVinci-Roboters speziell für Kopf-Hals-Eingriffe nicht im wirtschaftlichen Fokus des Herstellers liegt.

\section{Risiken}

In der Literatur erscheinen immer wieder einzelne Fallberichte, in denen der Bruch eines Instrumentenarms während eines Roboterassistierten Eingriffs beschrieben wird [82]. Diese Publikationen verdeutlichen, dass der Einsatz von Robotern neue Risiken für den Patienten birgt, welche in dem präoperativen Aufklärungsgespräch 
diskutiert werden müssen. Die einwirkenden Kräfte eines RoboterSystems sind z. T. erheblich. So konnte die Auflast, welche durch das Endoskop eines DaVinci ${ }^{\circledR}$ Systems generiert werden kann, in einer in vitro Messung auf über $7 \mathrm{~kg}$ beziffert werden [52]. Allerdings ist die Fraktur von menschlichen Knochen im Kopf-Hals-Bereich mit dem DaVinci-System laut Hockstein et al. auch mit Absicht im Kadaverversuch nicht möglich [61].

Beschreibungen von Komplikationen im Zusammenhang mit Roboter-Operationen werden entweder mittels Durchsicht der eigenen Patienten-Kohorten oder durch das systematische Aufarbeiten von Datenbanken generiert, welche entsprechende Einträge vorhalten. Eine ausführliche Übersichtsarbeit, die ihre Informationen aus der amerikanischen MAUDE (Manufacturer and User Facility Device Experience) Datenbank erhält, wurde kürzlich von Alemzadeh et al. publiziert [3]. Die automatische Datenbank-Recherche konnte für den Zeitraum von 2000 bis 2013 insgesamt 10624 Roboter-assoziierte Komplikationen bei ca. 1745000 Roboter-Eingriffen in den USA für alle Fachrichtungen identifizieren. Somit ergab sich eine Komplikationsquote von ca. 0.6\%. Die aufgelisteten Komplikationen beinhalteten unter anderem Verletzungen des Patienten ( $n=1391)$, Todesfälle ( $n=144)$ und Systemfehler $(n=8061)$. Über den untersuchten Zeitraum gesehen stieg die absolute Zahl der Komplikationen in Einklang mit der steigenden Anzahl der Roboter-Eingriffe kontinuierlich an. Allerdings überschritt auch die Komplikationsquote im Jahr 2013 erstmals die Marke von 1\%. Die Quote der Verletzungen und Todesfälle zeigte sich seit 2007 mit durchschnittlich $0.083 \%$ relativ stabil.

Interessanterweise war die Anzahl der Todesfälle verglichen mit der absoluten Anzahl von dokumentierten Komplikationen gerade für die Kopf-Hals-Chirurgie mit Abstand am höchsten drei (19,7\%, - Abb. 11). Dies ist wohl neben der anatomischen Komplexität des Halsbereichs auch dem abweichenden Dokumentationsverhalten unserer Fachrichtung geschuldet und beleuchtet einen wesentlichen Nachteil der Datenbank-basierten Auswertung. Weiterhin erfolgte die Berechnung der Quote von Roboter-Eingriffen mit tödlichem Ausgang im Verhältnis zu allen Roboter-assistierten Operationen. Diese war für Eingriffe in den Fachbereichen Herz-Thorax-Chirurgie und Kopf-Hals-Chirurgie 10-mal höher als in den Fachbereichen Gynäkologie, Urologie und Allgemeinchirurgie (0,052 \% vs. 0,0057\%; $p<0,001)$. Die Ursachen für die Todesfälle lagen fachübergreifend zu 75\% (64/86) im postoperativen Zeitraum, beinhalteten Sepsis und Blutungen und waren somit nicht direkt Roboter-assoziiert. Nur $17 \%$ der Todesfälle traten intraoperativ auf (15/86) und waren durch die unbeabsichtigte Verletzung von Organen (5/86), Blutung, Lungenembolie oder Herzstillstand verursacht. Sieben weitere Fälle konnten zeitlich nicht zugeordnet werden.

Ein Großteil (88\%) aller gemeldeten Komplikationen basierte auf Fehlern des Roboter-Systems und konnte unterteilt werden in Software-Fehler und Bildgebungsfehler (7,4\% aller Meldungen), Abbrechen von Geräteteilen in den Situs (14,7\%), Funkenschlag (10,5\%) sowie unbeabsichtigte Instrumentenbewegung (10,1\%). In Zusammenschau aller Meldungen führten die Komplikationen in 3,1 \% zum System-Neustart, in 7,3\% zur Umwandlung in eine nicht-robotische Operation und in 2,5\% zu einer Verlegung des Operationstermins.

Verletzungen und Todesfälle von Patienten haben oft ihre Ursache in einer Verkettung von mehreren Komplikationen, und ein einzelner Grund kann oft nicht identifiziert werden. Menschliches Fehl- verhalten und Fehlentscheidungen spielen allerdings laut MAUDEDatenbank eine entscheidende Rolle. Die häufigsten menschlichen Fehler werden wie folgt aufgelistet: Unzureichende Erfahrung in der Notfallbehandlung, unzureichendes Training am eingesetzten Roboter-System, unzureichende Überprüfung des Systems und der Instrumente vor dem Eingriff, unzureichende Lösung von bekannten technischen Problemen, inkorrektes Platzieren der Instrumente, inkorrekte Einstellung der Elektrokaustik, inkorrekte Kabelverbindungen, falsche Koordination von Hand- und Fußbewegungen, inkorrekter Wechsel der Instrumente.

Um die beschriebenen Komplikationen zu vermeiden, werden folgende Lösungsvorschläge angeboten: (1) Simulations-Umgebungen, in denen der Umgang mit Komplikationen ausreichend in einer sicheren Umgebung trainiert werden kann; (2) Intraoperative Echtzeit-Rückmeldung an den Operateur über sichere Operationspfade und anatomische Grenzen; (3) Verbesserte Dokumentationsmechanismen, welche im Falle von Komplikationen zur anschließenden Verbesserung des Systems beitragen können.

Trotz aller technischen Risiken, welche mit dem Einsatz von Roboter-Systemen neu auftreten, bleibt die postoperative Blutung die häufigste Komplikation bei Eingriffen im Kopf-Hals-Bereich. So konnte Zenga et al. in einem großen Patientenkollektiv ( $n=509)$ eine Nachblutungsrate von $3 \%$ dokumentieren, welche einer operativen Revision bedurfte [199]. Die Mehrheit der Patienten hatte die Diagnose eines Kopf-Hals-Karzinoms (75\%), andere waren aufgrund von nicht-malignen Pathologien im Kopf-Hals-Bereich operiert worden. Eine niedrigere Nachblutungsrate von 1,7\% zeigte sich in einem Kollektiv von Patienten, welche ausschließlich an obstruktiver Schlafapnoe litten und eine Zungengrundresektion erhalten hatten ( $n=243$ ) [183]. Zwei simultan publizierte Studien suggerieren, dass die Unterbindung der A. carotis externa bei TORS-Eingriffen nicht die Häufigkeit, aber die Intensität der postoperativen Nachblutung reduzieren kann $[57,87]$. Diese Aussage ist gut nachvollziehbar. Viel überraschender ist aber die hohe Nachblutungsrate mit operativer Revision, welche ohne Gefäßunterbindung bei jeweils 5,5\% ( $n=201)$ und 7,8\% $(n=265)$ lag. Pollei et al. haben die Nachblutungsrate bei TORS-Eingriffen und TLM-Eingriffen in einer retrospektiven, nichtrandomisierten Studie verglichen $(n=906)$ [143]. Hierbei zeigten sich ähnliche Nachblutungsraten für TORS (5,9\%) und TLM (5,6\%). Allerdings wurden größere Karzinome mit höherem T-Stadium signifikant öfter mit TLM als mit TORS operiert, sodass in dieser Studie von einem höheren Blutungsrisiko für TORS in vergleichbaren Tumorstadien ausgegangen werden muss. Zu demselben Ergebnis kommt eine französische, retrospektive Studie in Patienten mit Oropharynx-Karzinom ( $n=514)$, welche neben TORS ein höheres Alter und eine antikoagulative Therapie als signifikante Risikofaktoren für eine postoperative Nachblutung identifizieren konnten [91]. In einer weiteren monozentrischen, retrospektiven Übersichtsarbeit konnten Hay et al. eine Reduktion der TORS-assoziierten Komplikationen mit zunehmender Erfahrung des Operationsteams feststellen $(n=122)$ [60]. So wurden die schweren Komplikationen, wie Aspiration und Blutungen, über einen Zeitraum von 5 Jahren von 33 auf $10 \%$ reduziert.

Obwohl die hygienischen Bedenken bei der Aufbereitung der Roboter-Systeme weiter in den Fokus kommen, existieren hierzu bisher keine wissenschaftlichen Publikationen. Bei dem DaVinci-System sind besonders die kleineren Instrumente mit 5 mm Durchmesser 
betroffen, welche nach der Reinigung und Sterilisation nach Standardprotokoll weiter Gewebereste zwischen den Bauteilen aufweisen können. Um die aufwändigen Reinigungsverfahren zu umgehen, hat sich die Firma Medrobotics beim FLEX ${ }^{\circledR}$-System entschieden, Einmal-Instrumente zu verwenden, welche entsprechend teurer sind.

\section{Klinische Studien}

Die oben beschriebenen klinischen Vorteile der Roboter-assistierten Chirurgie im Kopf-Hals-Bereich können nur mittels klinischer Studien sinnvoll bestätigt werden. In ₹ Tab. 1 sind die aktuellen Studien aufgelistet, wobei der geringe Anteil an prospektiv randomisierten Studien auffällig ist. Die Daten entstammen der öffentlich zugänglichen NIH-Datenbank (www.clinicaltrials.gov) und erheben keinen Anspruch auf Vollständigkeit.

Bereits publiziert ist eine retrospektive, mehrarmige Studie in der TORS mit offener Chirurgie bei T1/T2 Malignomen des Oropharynx verglichen wird (S1, NCT00473564, n=36) [41]. Untersucht wurde hierbei die Länge des Krankenhausaufenthaltes, sowie die Dauer der Versorgung mit Tracheostoma und Ernährungssonde. TORS zeigte jeweils die besseren Ergebnisse, wobei aufgrund des retrospektiven Studienaufbaus von einem Bias zugunsten von TORS bei der Patientenselektion ausgegangen werden muss. Ebenso publiziert ist die prospektive, einarmige Zulassungsstudie für das oben beschriebene Flex ${ }^{\circledR}$-System der Firma Medrobotics (S2, NCT02262247, n = 80) [94]. In dieser mehrheitlich nicht-onkologischen Patientenkohorte konnte der sichere und effektive Einsatz des Systems im Pharynx und der Supraglottis nachgewiesen werden. Zwei weitere Studien untersuchen die Durchführbarkeit von TORS-Eingriffen im Pharynx und Larynx. Neben der Durchführbarkeit werden auch die Effektivität und die Lebensqualität im Langzeitverlauf ausgewertet. Die Rekrutierungsphase der ersten Phase I Studie ist bereits abgeschlossen, die Daten sind allerdings noch nicht publiziert (S3, NCT01059357, $\mathrm{n}=60$ ). Die zweite Phase I Studie ist aktuell geöffnet (S4, NCT0181 $9480, n=85$ ). Eine weitere amerikanische, monozentrische, einarmige Studie untersucht neben dem onkologischen Resultat in Patienten mit Oropharynx-Karzinom auch die Schluck- und Sprechfähigkeit nach TORS (S5, NCT02225496, n=140). Die größte, nichtrandomisierte, einarmige Studie zu TORS stammt ebenso aus den USA und umfasst Patienten mit malignen und benignen Pathologien zum Nachweis der Durchführbarkeit von TORS (S6, NCT01473784, $\mathrm{n}=360$ ).

Mit dem besonderen Wachstumsverhalten von $\mathrm{HPV}^{+}$Tumoren beschäftigen sich 2 aktuell laufende einarmige Studien aus den USA. In TORS-behandelten Patienten untersuchen Lin et al., ob die Region des Primärtumors bei der adjuvanten Radiatio ausgespart werden kann ohne eine Reduktion des Überlebens zu riskieren (S7, NCT02159703, n=60). Die SIRS-Studie evaluiert die Möglichkeit zur De-Eskalation der adjuvanten Therapie in TORS-behandelten, HPV + Patienten. Konnte der Primärtumor operativ vollständig entfernt werden und liegen nicht mehr als 2 tumorinfiltrierte Lymphknoten vor, so wird auf die adjuvante Radiatio zunächst verzichtet und die Kontrolle mittels PET-CT durchgeführt (S8, NCT02072148, n=200).

Den Stellenwert der Induktions-Chemotherapie mit Cisplatin, Paclitaxel und Nivolumab untersucht die OPTIMA-II Studie von Siewert et al. (S9, NCT03107182, n=56). Bei ausreichendem Ansprechen des Tumors erfolgt nach der Induktionsphase die Roboter-assistier- te Tumorexzision. Alle anderen Patienten erhalten eine kombinierte Radiochemotherapie. Ein ähnliches Konzept bietet die Studie von Sadeghi et al., bei der die Induktionschemotherapie mit Cisplatin und Doxetacel durchgeführt wird (S10, NCT02760667, n=20). Die anschließende Tumortherapie wird entweder mittels TORS oder mit transoraler Laser-Mikrochirurgie durchgeführt.

In Patienten mit obstruktivem Schlafapnoesyndrom (OSAS) konnte eine bereits publizierte einarmige Phase I Studie zeigen, dass die Kombination aus Roboter-assistierter Zungengrundreduktion und Uvulopalatopharyngoplastik (UPPP) auch für bereits vor-operierte Patienten einen klinischen Vorteil bringen kann (S11, NCT01187160, $\mathrm{n}=75$ ) [175]. Eine ähnliche Studie zur TORS-Therapie bei OSAS-Patienten befindet sich aktuell in der Rekrutierungsphase (S12, NCT01107795, $n=75$ ).

Eine Serie von einarmigen klinischen Studien versucht, neue Indikationsfelder für TORS zu erschließen. Aufgrund der jeweils neuartigen Ansätze sind die Patientenzahlen in diesen Pilotstudien sehr limitiert. So wird in einer sehr kleinen französischen Studie der transaxilläre Zugang zur Neck dissection (Level II-IV) untersucht, welcher aufgrund der fehlenden zervikalen Narbe kosmetisch vorteilhaft erscheint (S13, NCT02269020, n=3). Das freie Muskeltransplantat des M. latissimus dorsi wird oft in der rekonstruktiven Chirurgie verwendet. Das Roboter-gestützte Heben des Muskeltransplantats wird in einer monozentrischen Pilotstudie evaluiert (S14, NCT02274493, $\mathrm{n}=15$ ). Der Zugang zur Hypophyse über die Sella turcica mittels TORS ist von einer französischen Arbeitsgruppe in einer Kadaverstudie publiziert worden [32]. Die anschließende klinische Studie, in welcher der Zugang zur Sella turcica mit den Manipulator-Armen eines DaVinci Si ${ }^{\circledR}$ demonstriert werden soll, befindet sich in der Rekrutierungsphase (S15, NCT02743442, $n=8$ ). Moore et al. untersuchen im Rahmen einer Pilotstudie die Möglichkeit, TORS-Eingriffe in sitzender Position durchzuführen (S16, NCT02792322, n=50). Die Autoren versprechen sich hierdurch eine verbesserte Visualisierung des Operationsgebiets und somit eine kürzere Operationsdauer, wie bereits in 2 Patienten durchgeführt [123]. Die technischen Weiterentwicklungen des DaVinci ${ }^{\circledR}$ Systems werden in 2 unabhängigen Studien evaluiert. Eine französische Gruppe untersucht die Vorteile des DaVinci ${ }^{\circledR}$ Xi in einer einarmigen Phase I Studie für TORS (S17, NCT02517125, $n=150$ ). Simon et al. testen ein neuartiges Single Port System für den Einsatz bei TORS, aber auch bei kolorektalen und urologischen Indikationen (S18, NCT03010813, n=60). Laut Berichten des Internet-Blogs „www.surgrob.blogspot.com“ hat Intuitive Surgical 10 Prototypen des DaVinci SP ${ }^{\circledR}$ (single port) produziert, welche aktuell im klinischen Einsatz getestet werden. Bei letztgenannter Studie könnte es sich daher um einen dieser Prototypen handeln.

Bereits publiziert ist die nicht-randomisierte südkoreanische Studie zur Neck dissection bei cN0, welche den modifizierten Roboterassistierten face-lift Zugang mit dem konventionellen offenen Zugang vergleicht (S19, NCT01488669, $n=26$ ). Die Operationsdauer war in der TORS-Gruppe etwa doppelt so lang (78 vs. $158 \mathrm{~min}$ ). Die Anzahl der durchschnittlich entnommenen Lymphknoten war allerdings vergleichbar (20 vs. 22), und das kosmetische Resultat in der TORS-Gruppe besser [97].

Unzweifelhaft sind die vielen publizierten Fallserien und nichtrandomisierten Pilotstudien von gewisser Bedeutung für die technische Weiterentwicklung und Erweiterung der operativen Ansätze. Wissenschaftlich besonders aussagekräftig sind aber mit Sicherheit 


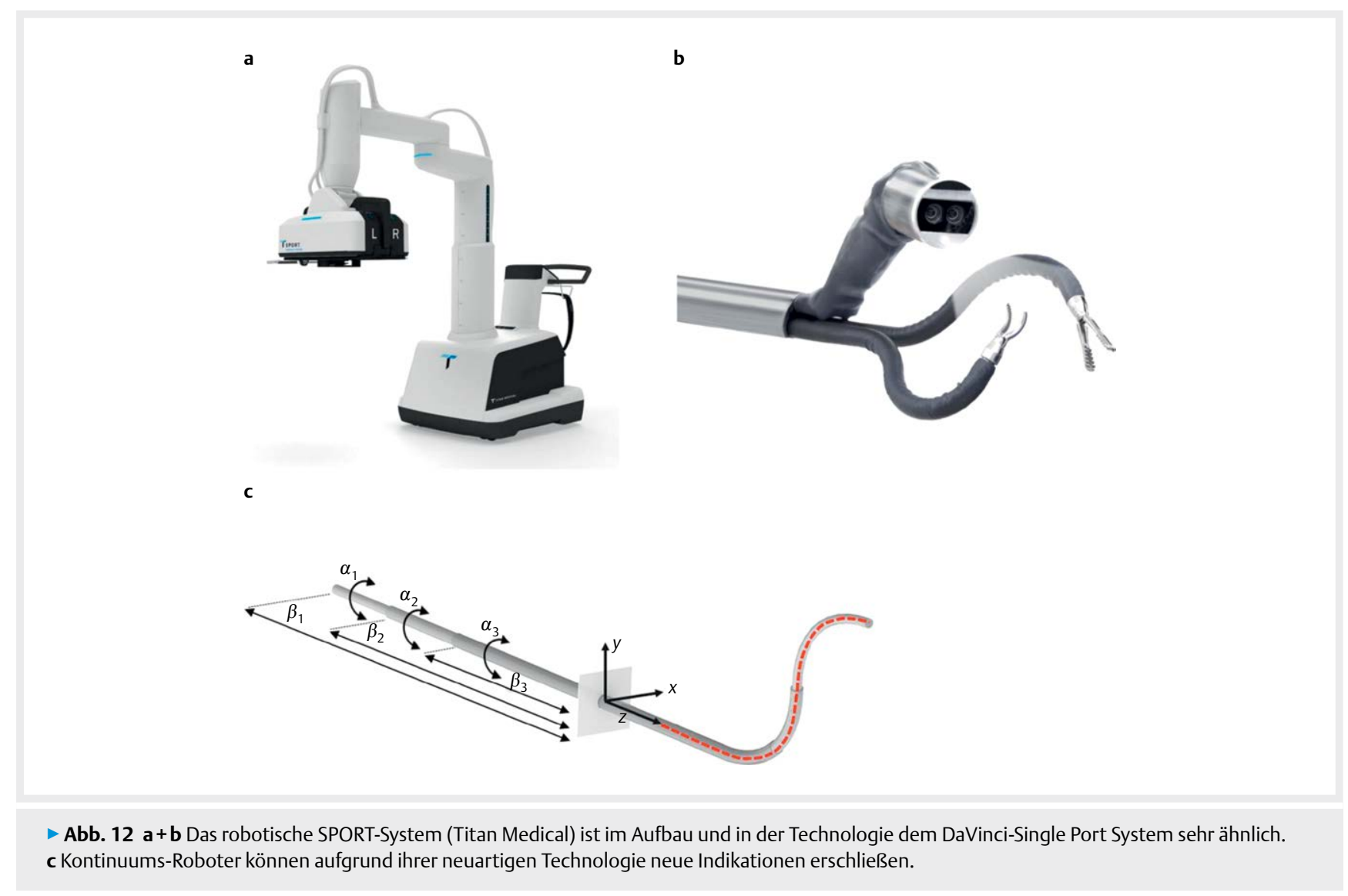

die folgenden prospektiven, randomisierten klinischen Studien. Der vielfach höhere Organisationsaufwand und die deutlich erschwerte Patientenrekrutierung dürfen hierbei nicht unterschätzt werden. Die interventionelle Phase-II Studie von Ferris et al. erprobt die Deeskalation der adjuvanten Radiatio in HPV + Patienten nach operativer Tumorexzision mittels TORS (S20, NCT01898494, n=377). Die Intensitätsmodulierte Radiatio (IMRT) wird nach der Operation zwischen der Standard-Dosis (60 Gy) und einer erniedrigten Dosis (50 Gy) randomisiert [65]. Die Kombination von TORS mit einer neoadjuvanten Immuntherapie mittels Tumor-Vakzinierung wird in der Arbeitsgruppe von Sikora et al. evaluiert (S21, NCT02002182, $n=30$ ). Die intravenöse Lebend-Impfung wird mit genetisch modifizierten Listerien durchgeführt, welche das HPV-spezifische Onkoprotein E7 exprimieren [35]. Nach der Impfung erhalten alle eingeschlossenen Patienten eine TORS-basierte Tumorexstirpation. Während die beiden erstgenannten Studien die adjuvante Therapie vor oder nach TORS randomisieren, sind die folgenden Studien speziell dafür ausgelegt, den Einsatz von TORS gegenüber der Standardtherapie zu vergleichen.

Eine extrem große brasilianische randomisierte Phase-III Studie vergleicht die Roboter-assistierte Operation mit der Standard-Operation für verschiedene Tumorentitäten aus allen Fachbereichen (S22, NCT02292914, n=1120). Der gemeinsame europäische Ansatz der EORTC (European Organisation for Reseach an Treatment of Cancer) unterscheidet nicht zwischen den verschiedenen operativen Verfahren, wie TORS oder TLM, wenn diese mit der konservativen Therapie mittels Radiatio randomisiert verglichen werden (S23, NCT02984410, n=170). Eingeschlossen werden Patienten mit frü- hem Oropharynx-Karzinom (T1, T2), welche sich für chirurgische und konservative Therapieverfahren qualifizieren. Den selben Ansatz verfolgt die kanadische ORATOR-Studie ebenfalls beim frühen Oropharynx-Karzinom (S24, NCT01590355, n=68). Allerdings ist hier TORS als alleinige operative Option vorgesehen [127]. Diese Studien versprechen in den nächsten Jahren höchst interessante Resultate, was den Vergleich von operativen Ansätzen inklusive TORS mit konservativen Therapiemethoden betrifft.

Abschließend wird eine Studie beschrieben, welche die Lebensqualität im Zusammenhang mit TORS-Therapie evaluiert. Gross et al. vergleichen in einer nicht-randomisierten, prospektiven Studie den Aktivitätsgrad von Patienten nach TORS oder nach konservativer Tumortherapie (S25, NCT02663583, n=44). Die Aktivität des Patienten wird mithilfe eines elektronischen Armbandes gemessen, welches die Bewegungen des Patienten aufzeichnet.

\section{Neue Technologien}

Bekannterweise ist das Potenzial der Roboter-assistierten Chirurgie enorm. Da aber gleichzeitig die hohen Erwartungen bisher noch nicht erfüllt werden konnten, lebt die Roboter-assistierte Chirurgie von der ständigen Weiterentwicklung und Spezialisierung. Im folgenden Abschnitt werden technische Weiterentwicklungen präsentiert, welche noch nicht in der klinischen Routine präsent sind, die Chirurgie in naher Zukunft möglicherweise aber mitgestalten werden.

Neben den zugelassenen Roboter-Systemen DaVinci ${ }^{\circledR}$ und FLEX ${ }^{\circledR}$ existieren zahlreiche weitere Systeme, welche sich aktuell in der präklinischen Erprobungsphase befinden. Im Rahmen eines EU-gespon- 
sorten Forschungsprojektes wurde das MicroRALP-System entwickelt [114]. Dieses teilflexible Endoskop wird transoral eingeführt und ermöglicht eine 3D-Visualisierung der Stimmlippen. Über einen interaktiven Bildschirm gibt der Operateur die Grenzen für die Resektion vor, welche anschließend halbautomatisch durch den integrierten Laserstrahl durchgeführt wird. Weitere Informationen finden sich auf der Projektseite im Internet unter www.microralp.eu. Das System konnte bisher in Kadaverexperimenten getestet werden und befindet sich zur Zeit in der Weiterentwicklung.

Die Arbeitsgruppe um Olds et al. hat in der Vergangenheit ein flexibles Endoskop entwickelt, welches einhändig über eine Joystick gesteuert werden kann [134]. Dieses Robo-ELF System wurde speziell für die Larynxchirurgie entwickelt und gibt dem Operateur die Möglichkeit mit der freien Hand ein zusätzliches Instrument zu bedienen [133]. Robo-ELF wurde bisher in Kadaverexperimenten getestet, über die Weiterentwicklung existieren aktuell keine Informationen.

Ein Single-Port-System mit dem Namen ,SPORT ' der Firma Titan Medical ${ }^{\circledR}$ ist bereits erfolgreich für die Abdominalchirurgie in lebenden Tieren getestet worden. Entsprechende wissenschaftliche Publikationen liegen allerdings nicht vor. Neben 2 flexiblen Armen besitzt das System einen flexiblen Kamerakopf und erinnert somit an die neueste Single-Port-Version des DaVinci-Systems. Alle 3 Arme können nach Einführen in den Körper trianguliert werden, um eine effiziente Arbeitshaltung zu erreichen ( $\triangleright \mathbf{A b b}$. 12a/b). Es ist durchaus denkbar, dass diese Konstruktion auch Vorteile für die Kopf-HalsChirurgie bringt, wenn die Ausmaße der Instrumente entsprechend angepasst werden.

Interessant ist auch die offene Forschungsplattform, welche in USA konstruiert worden ist. Die Plattform besteht aus Komponenten ausgedienter DaVinci-Systeme und bietet über eine Linux-Software unbegrenzt Zugang zu der gesamten Software und Elektronik [75]. Aktuell sind mindestens 11 Plattformen an verschiedenen Standorten installiert.

Kontinuums-Roboter bestehen aus mehreren konzentrischen Röhren, welche ineinander verschachtelt werden [21]. Jede Röhre kann mit elektrischen Motoren unabhängig voneinander bewegt werden, womit ein 3-dimensionaler Raum vollständig abgedeckt wird ( $>$ Abb. 12c). Die logistische Denkarbeit, wie die Röhren zu einander stehen müssen, wird von einem Rechner übernommen. Dieses Bewegungsmodell unterscheidet sich vollständig von den bisherigen Roboter-Systemen und könnte dank seiner nicht-linearen Bauweise besonders für die Nasennebenhöhlen- und Schädelbasischirurgie von Interesse sein. Mehrere Arbeitsgruppen befassen sich mit der Entwicklung von Kontinuums-Robotern, wobei sich Wu et al. und BurgnerKahrs et al. auch mit den Herausforderungen im HNO-Bereich beschäftigen $[23,196]$.

Auch in der Roboter-assistierten Chirurgie ist der Einsatz von Lasern zur Geweberesektion wünschenswert. Allerdings ist die dauerhafte Fokussierung des Laserstrahls mithilfe der Roboterinstrumente eine bisher ungelöste Herausforderung [170]. Zusätzlich fallen die Kosten für eine flexible Laserfaser zusätzlich ins Gewicht, welche bei der klassischen mikroskopischen Laserresektion nicht auftreten [64]. Üblicherweise werden in der Kopf-Hals-Chirurgie $\mathrm{CO}_{2}$-Laser für die Resektion verwendet [112]. Alternative Laserquellen, wie der Erbium-YAG-Laser zeigen in experimentellen Ansätzen qualitativ bessere Schneide-Eigenschaften und bessere Möglichkeiten zur Fokussierung [169]. Somit sind diese Laserquellen möglicherweise besser geeignet für den Einsatz in der Roboter-assistierten Chirurgie.

Eine weitere Informationsquelle für den Operateur kann speziell in der Larynxchirurgie die Endosonografie dienen [84]. Hierfür wird ein spezieller Ultraschallkopf in den mit Wasser gefüllten Larynx eingeführt, um zusätzliche anatomische Details zu erkennen.

Die Roboter-assistierte Chirurgie bietet besonders aufgrund der elektronisch transportierten Bildgebung neue Möglichkeiten zum Einsatz von Software, welche den Operateur in seiner Arbeit unterstützt. Allgemein bekannt sind die Vorteile des ,narrow band imaging für die frühzeitige Erkennung von Präkanzerosen oder die bessere Abgrenzung von malignen Strukturen [83]. Darüber hinaus bestehen aber zusätzliche Technologien, wie die Hochfrequenz-Bildgebung und optische Kohärenztomografie [176]. In dem oben genannten MicroRALPProjekt sind multiple Software-Lösungen zum Einsatz gekommen. Diese ermöglichen z. B. die automatische Verfolgung von beweglichen Gewebestrukturen (soft tissue tracking) [157], Methoden zur Oberflächenrekonstruktion [156] oder die automatische Instrumentenerkennung durch das Roboter-System [5]. All diesen Methoden könnte die Roboter-assistierte Chirurgie den Weg in die routinemäßige Anwendung eröffnen.

Abschließend werden auch geografische Distanzen überwunden und Roboter-assistierte Operationen können über große Entfernungen hinweg durchgeführt werden. So konnte im Tiermodell schon eine Gallenblasen-Resektion durchgeführt werden, bei welcher sich der Operateur in New York und der Patient im 7000 Kilometer entfernten Straßburg befand [111].

\section{Schlussfolgerung}

Mit steigenden Fallzahlen und der Erweiterung der Indikationen wird das Potenzial der RAC-Technologie im Kopf-Hals-Bereich deutlich. Mit Sicherheit werden einige Patientengruppen in Zukunft davon profitieren können. Einige fokussierte Zentren verwenden RoboterSysteme schon heute regelmäßig für Eingriffe im Oropharynx-Bereich oder an der Schilddrüse. Rein experimentell verbleibt aktuell die Anwendung der RAC an der Schädelbasis und den Nasennebenhöhlen. Trotz aller Fortschritte ist der sichere Nachweis eines Vorteils der RAC gegenüber den konventionellen Operationsmethoden wie transorale Lasermikrochirurgie bisher nicht erbracht worden. Zusätzlich stellen sicherlich der finanzielle Mehraufwand und die weiterhin aufwändige Handhabung der Roboter-Systeme die größten Hürden für einen flächendeckenden Einsatz der RAC in der klinischen Routine dar. Weitere randomisierte klinische Studien können helfen, um die Vor- und Nachteile dieser spannenden Technologie weiter zu beleuchten.

Wünschenswert sind außerdem spezielle Instrumente und auch alternative RAC-Systeme für die wachsende Anzahl an möglichen RAC-Indikationen. Ebenso ist eine Kombination mit anderen technischen Weiterentwicklungen, wie z. B. hyperspektrale Bildgebung, visuelle Haptik oder automatisierte Resektionsmechanismen, möglich.

Zusammenfassend kann die RAC die bisherigen konventionellen Operationsmethoden in Zukunft sinnvoll ergänzen ohne diese vollständig abzulösen. Technische, wissenschaftliche und ethische Konflikte werden daher die Arbeit der aktuellen Generation von KopfHals-Chirurgen im zunehmenden Maße beeinflussen. 
Interessenkonflikt

Der Autor hat keine finanzielle Unterstützung für die Autorenschaft oder Publikation dieses Artikels erhalten. Die Arbeitsgruppe des Autors hat Kadaverstudien in den Räumlichkeiten der Firma Medrobotics (USA) durchgeführt, für welche die Reisekosten übernommen worden sind, und hat an der klinischen Zulassungsstudie der Firma Medrobotics (NCT02262247) teilgenommen. Während der Studie wurde ein Unkostenbeitrag pro Fall an die Firma bezahlt. Weiterhin hat die Arbeitsgruppe des Autors Kadaverstudien durchgeführt, welche von der Firma Medineering (München) finanziell unterstützt worden sind.

Literatur

[1] Al Kadah B, Piccoli M, Mullineris B et al. Modifications of transaxillary approach in endoscopic da Vinci-assisted thyroid and parathyroid gland surgery. J Robot Surg 2015; 9: 37-44

[2] Albus JS. NBS/RIA Robotics Research Workshop: proceedings of the NBS/RIA Workshop on Robotic Research held at Gaithersburg, MD. November 13-15, 1979/sponsored by The Robot Institute of America. Washington: National Bureau of Standards. 1979

[3] Alemzadeh H, Raman J, Leveson N et al. Adverse Events in Robotic Surgery: A Retrospective Study of 14 Years of FDA Data. PLoS One 2016; 11: e0151470

[4] Ali SM, Reisner LA, King B et al. Eye gaze tracking for endoscopic camera positioning: an application of a hardware/software interface developed to automate Aesop. Stud Health Technol Inform 2008; 132: 4-7

[5] Allan M, Ourselin S, Thompson S et al. Toward detection and localization of instruments in minimally invasive surgery. IEEE Trans Biomed Eng 2013; 60: 1050-1058

[6] Antoniou GA, Riga CV, Mayer EK et al. Clinical applications of robotic technology in vascular and endovascular surgery. J Vasc Surg 2011; 53: 493-499

[7] Arshad H, Durmus K, Ozer E. Transoral robotic resection of selected parapharyngeal space tumors. Eur Arch Otorhinolaryngol 2013; 270: 1737-1740

[8] Aubry K, Vergez S, De Mones E et al. Morbidity and mortality revue of the French group of transoral robotic surgery: a multicentric study. J Robot Surg 2016; 10: 63-67

[9] Ban E], Yoo JY, Kim WW et al. Surgical complications after robotic thyroidectomy for thyroid carcinoma: a single center experience with 3,000 patients. Surg Endosc 2014; 28: 2555-2563

[10] Benali-Khoudja M, Hafez M, Alexandre JM et al. Tactile interfaces: a state-of-the-art survey. Proc. Int. Symp. Robot 2004; 31: 23-26

[11] Benhidjeb T, Wilhelm T, Harlaar J et al. Natural orifice surgery on thyroid gland: totally transoral video-assisted thyroidectomy (TOVAT): report of first experimental results of a new surgical method. Surg Endosc 2009; 23: 1119-1120

[12] Bethea BT, Okamura AM, Kitagawa M et al. Application of haptic feedback to robotic surgery. J Laparoendosc Adv Surg Tech A 2004; 14: 191-195

[13] Biron VL, O'connell DA, Barber B et al. Transoral robotic surgery with radial forearm free flap reconstruction: case control analysis. J Otolaryngol Head Neck Surg 2017; 46: 20

[14] Blanco RG, Ha PK, Califano JA et al. Robotic-assisted neck dissection through a pre- and post-auricular hairline incision: preclinical study. J Laparoendosc Adv Surg Tech A 2012; 22: 791-796

[15] Blanco RG, Ha PK, Califano JA et al. Transoral robotic surgery of the vocal cord. J Laparoendosc Adv Surg Tech A 2011; 21: 157-159
[16] Blavier A, Gaudissart Q, Cadiere GB et al. Impact of 2D and 3D vision on performance of novice subjects using da Vinci robotic system. Acta Chir Belg 2006; 106: 662-664

[17] Bolenz C, Freedland S], Hollenbeck BK et al. Costs of radical prostatectomy for prostate cancer: a systematic review. Eur Urol 2014; 65: 316-324

[18] Bonawitz SC, Duvvuri U. Robot-assisted oropharyngeal reconstruction with free tissue transfer. J Reconstr Microsurg 2012; 28: 485-490

[19] Bowyer SA, Davies BL, Rodriguez F. Active constraints/virtual fixures: A survey. IEEE Trans Robot 2014; 30: 138-157

[20] Bumm K, Wurm J, Rachinger J et al. An automated robotic approach with redundant navigation for minimal invasive extended transsphenoidal skull base surgery. Minim Invasive Neurosurg 2005; 48: 159-164

[21] Burgner-Kahrs ], Rucker DC, Choset H. Continuum Robots for Medical Applications: A Survey. IEEE Trans Robot 2015; 31: 1261-1280

[22] Burgner J, Rucker DC, Gilbert HB et al. A Telerobotic System for Transnasal Surgery. IEEE ASME Trans Mechatron 2013; 19: 996-1006

[23] Burgner J, Swaney PJ, Lathrop RA et al. Debulking from within: a robotic steerable cannula for intracerebral hemorrhage evacuation. IEEE Trans Biomed Eng 2013; 60: 2567-2575

[24] Byeon HK, Duvvuri U, Kim WS et al. Transoral robotic retropharyngeal lymph node dissection with or without lateral oropharyngectomy. J Craniofac Surg 2013; 24: 1156-1161

[25] Byeon HK, Holsinger FC, Tufano RP et al. Robotic total thyroidectomy with modified radical neck dissection via unilateral retroauricular approach. Ann Surg Oncol 2014; 21: 3872-3875

[26] Byeon HK, Kim Da H, Chang JW et al. Comprehensive application of robotic retroauricular thyroidectomy: The evolution of robotic thyroidectomy. Laryngoscope 2016; 126: 1952-1957

[27] Byrd JK, Smith KJ, De Almeida JR et al. Transoral Robotic Surgery and the Unknown Primary: A Cost-Effectiveness Analysis. Otolaryngol Head Neck Surg 2014; 150: 976-982

[28] Carrau RL, Prevedello DM, De Lara D et al. Combined transoral robotic surgery and endoscopic endonasal approach for the resection of extensive malignancies of the skull base. Head Neck 2013; 35: E351-E358

[29] Castelnuovo P, Nicolai P, Turri-Zanoni M et al. Endoscopic endonasal nasopharyngectomy in selected cancers. Otolaryngol Head Neck Surg 2013; 149: 424-430

[30] Chai Y], Lee KE, Youn YK. Can robotic thyroidectomy be performed safely in thyroid carcinoma patients? Endocrinol Metab (Seoul) 2014; 29: $226-232$

[31] Chan JYW, Chan RCL, Chow VLY et al. Transoral robotic total laryngopharyngectomy and free jejunal flap reconstruction for hypopharyngeal cancer. Oral Oncol 2017; 72: 194-196

[32] Chauvet D, Missistrano A, Hivelin M et al. Transoral robotic-assisted skull base surgery to approach the sella turcica: cadaveric study. Neurosurg Rev 2014; 37: 609-617

[33] Cho HJ, Kang JW, Min HJ et al. Robotic nasopharyngectomy via combined endonasal and transantral port: a preliminary cadaveric study. Laryngoscope 2015; 125: 1839-1843

[34] Chung TK, Rosenthal EL, Magnuson JS et al. Transoral robotic surgery for oropharyngeal and tongue cancer in the United States. Laryngoscope 2015; 125: 140-145

[35] Cory L, Chu C. ADXS-HPV: a therapeutic Listeria vaccination targeting cervical cancers expressing the HPV E7 antigen. Hum Vaccin Immunother 2014; 10: 3190-3195

[36] Dallan I, Castelnuovo P, Montevecchi F et al. Combined transoral transnasal robotic-assisted nasopharyngectomy: a cadaveric feasibility study. Eur Arch Otorhinolaryngol 2012; 269: 235-239 
[37] Dallan I, Castelnuovo P, Seccia V et al. Combined transnasal transcervical robotic dissection of posterior skull base: feasibility in a cadaveric model. Rhinology 2012; 50: 165-170

[38] De Almeida JR, Li R, Magnuson JS et al. Oncologic Outcomes After Transoral Robotic Surgery: A Multi-institutional Study. JAMA Otolaryngol Head Neck Surg 2015; 141: 1043-1051

[39] De Almeida JR, Moskowitz AJ, Miles BA et al. Cost-effectiveness of transoral robotic surgery versus (chemo)radiotherapy for early $T$ classification oropharyngeal carcinoma: A cost-utility analysis (ePub). Head Neck 2014

[40] De Almeida JR, Park RC, Genden EM. Reconstruction of transoral robotic surgery defects: principles and techniques. J Reconstr Microsurg 2012; 28: 465-472

[41] Dean NR, Rosenthal EL, Carroll WR et al. Robotic-assisted surgery for primary or recurrent oropharyngeal carcinoma. Arch Otolaryngol Head Neck Surg 2010; 136: 380-384

[42] Diaz I, Gil J], Louredo M. A haptic pedal for surgery assistance Comput Methods Programs Biomed 2014; 116: 97-104

[43] Dombree M, Crott R, Lawson G et al. Cost comparison of open approach, transoral laser microsurgery and transoral robotic surgery for partial and total laryngectomies. Eur Arch Otorhinolaryngol 2014; 271: $2825-2834$

[44] Duke WS, Holsinger FC, Kandil E et al. Remote Access Robotic Facelift Thyroidectomy: A Multi-institutional Experience. World J Surg 2017; 41: $116-121$

[45] Dziegielewski PT, Teknos TN, Durmus K et al. Transoral Robotic Surgery for Oropharyngeal Cancer: Long-term Quality of Life and Functional Outcomes. JAMA Otolaryngol Head Neck Surg 2013; 1-9

[46] Enayati N, De Momi E, Ferrigno G. Haptics in Robot-Assisted Surgery: Challenges and Benefits. IEEE Rev Biomed Eng 2016; 9: 49-65

[47] Farrag TY, Lin FR, Cummings CW et al. Neck management in patients undergoing postradiotherapy salvage laryngeal surgery for recurrent/ persistent laryngeal cancer. Laryngoscope 2006; 116: 1864-1866

[48] Fernandez-Fernandez MM, Gonzalez LM, Calvo CR et al. Transoral ultrasonic total laryngectomy (TOUSS-TL): description of a new endoscopic approach and report of two cases (ePub). Eur Arch Otorhinolaryngol 2016; 273: 2689-2696

[49] Friedman M, Hamilton C, Samuelson CG et al. Transoral robotic glossectomy for the treatment of obstructive sleep apnea-hypopnea syndrome. Otolaryngol Head Neck Surg 2012; 146: 854-862

[50] Friedrich DT, Scheithauer MO, Greve J et al. Potential Advantages of a Single-Port, Operator-Controlled Flexible Endoscope System for Transoral Surgery of the Larynx. Ann Otol Rhinol Laryngol 2015; 124 : 655-662

[51] Friedrich DT, Scheithauer MO, Greve J et al. Application of a computer-assisted flexible endoscope system for transoral surgery of the hypopharynx and upper esophagus. Eur Arch Otorhinolaryngol 2017; 274: 2287-2293

[52] Fujiwara K, Fukuhara T, Niimi K et al. Load evaluation of the da Vinci surgical system for transoral robotic surgery. J Robot Surg 2015; 9: 315-319

[53] Fung K, Teknos TN, Vandenberg CD et al. Prevention of wound complications following salvage laryngectomy using free vascularized tissue. Head Neck 2007; 29: 425-430

[54] Ganly I, Patel S, Matsuo J et al. Postoperative complications of salvage total laryngectomy. Cancer 2005; 103: 2073-2081

[55] Garas G, Kythreotou A, Georgalas C et al. Is transoral robotic surgery a safe and effective multilevel treatment for obstructive sleep apnoea in obese patients following failure of conventional treatment(s)? Ann Med Surg (Lond) 2017; 19: 55-61

[56] Genden EM, Park R, Smith C.et al. The role of reconstruction for transoral robotic pharyngectomy and concomitant neck dissection. Arch Otolaryngol Head Neck Surg 2011; 137: 151-156
[57] Gleysteen J, Troob S, Light T et al. The impact of prophylactic external carotid artery ligation on postoperative bleeding after transoral robotic surgery (TORS) for oropharyngeal squamous cell carcinoma. Oral Oncol 2017; 70: 1-6

[58] Golbin D, Musgrave B, Succar E et al. Clinical analysis of drug-induced sleep endoscopy for the OSA patient. Laryngoscope 2016; 126 : 249-253

[59] Hanna EY, Holsinger C, Demonte F et al. Robotic endoscopic surgery of the skull base: a novel surgical approach. Arch Otolaryngol Head Neck Surg 2007; 133: 1209-1214

[60] Hay A, Migliacci ], Karassawa Zanoni D et al. Complications following transoral robotic surgery (TORS): A detailed institutional review of complications. Oral Oncol 2017; 67: 160-166

[61] Hockstein NG, O'malley BW Jr., Weinstein GS. Assessment of intraoperative safety in transoral robotic surgery. Laryngoscope 2006; 116: 165-168

[62] Hoff PT, D’agostino MA, Thaler ER. Transoral robotic surgery in benign diseases including obstructive sleep apnea: Safety and feasibility. Laryngoscope 2015; 125: 1249-1253

[63] Hoffmann TK, Friedrich DT, Schuler P]. [Robot-assisted surgery in the head and neck region]. HNO 2016; 64: 658-666

[64] Hoffmann TK, Schuler PJ, Bankfalvi A et al. Comparative analysis of resection tools suited for transoral robot-assisted surgery. Eur Arch Otorhinolaryngol 2014; 271: 1207-1213

[65] Holsinger FC, Ferris RL. Transoral Endoscopic Head and Neck Surgery and Its Role Within the Multidisciplinary Treatment Paradigm of Oropharynx Cancer: Robotics, Lasers, and Clinical Trials. J Clin Oncol 2015; 33: 3285-3292

[66] Hurtuk AM, Marcinow A, Agrawal A et al. Quality-of-life outcomes in transoral robotic surgery. Otolaryngol Head Neck Surg 2012; 146: 68-73

[67] Intuitivesurgical Annual Report. http://www.annualreports.com/ Company/intuitive-surgical-inc 2016

[68] Ishikawa N, Kawaguchi M, Moriyama $\mathrm{H}$ et al. Robot-assisted thyroidectomy with novel camera-port retractor. Innovations (Phila) 2013; 8: 384-388

[69] Jenkins K, Nguyen V, Zhu R et al. Piezotronic Effect: An Emerging Mechanism for Sensing Applications. Sensors (Basel) 2015; 15: 22914-22940

[70] Kaczmar JM, Tan KS, Heitjan DF et al. HPV-related oropharyngeal cancer: Risk factors for treatment failure in patients managed with primary transoral robotic surgery. Head Neck 2016; 38: 59-65

[71] Kajiwara N, Patrick Barron J, Kato Y et al. Cost-Benefit Performance of Robotic Surgery Compared with Video-Assisted Thoracoscopic Surgery under the Japanese National Health Insurance System. Ann Thorac Cardiovasc Surg 2015; 21: 95-101

[72] Kang SW, Jeong JJ, Yun JS et al. Gasless endoscopic thyroidectomy using trans- axillary approach; surgical outcome of 581 patients. Endocr J 2009; 56: 361-369

[73] Kang SW, Lee SH, Ryu HR et al. Initial experience with robot-assisted modified radical neck dissection for the management of thyroid carcinoma with lateral neck node metastasis. Surgery 2010; 148 : 1214-1221

[74] Kayhan FT, Kaya KH, Sayin I. Transoral robotic cordectomy for early glottic carcinoma. Ann Otol Rhinol Laryngol 2012; 121: 497-502

[75] Kazanzides P, Chen Z, Deguet A. et al. An Open-Source Research Kit for the da Vinci Surgical System. 2014 IEEE International Conference on Robotics \& Automation (ICRA) 978-1-4799-3685-4/14/. 2014

[76] Kim U, Lee DH, Yoon W] et al. Force Sensor Integrated Surgical Forceps for Minimally Invasive Robotic Surgery. leee T Robot 2015; 31: 1214-1224 
[77] Kim WS, Ban MJ, Chang JW et al. Learning curve for robot-assisted neck dissection in head and neck cancer: a 3-year prospective case study and analysis. JAMA Otolaryngol Head Neck Surg 2014; 140: 1191-1197

[78] Kim WS, Byeon HK, Park YM et al. Therapeutic robot-assisted neck dissection via a retroauricular or modified facelift approach in head and neck cancer: a comparative study with conventional transcervical neck dissection. Head Neck 2015; 37: 249-254

[79] Kim WS, Koh YW, Byeon HK et al. Robot-assisted neck dissection via a transaxillary and retroauricular approach versus a conventional transcervical approach in papillary thyroid cancer with cervical lymph node metastases. J Laparoendosc Adv Surg Tech A 2014; 24 : 367-372

[80] Kim WS, Lee HS, Kang SM et al. Feasibility of robot-assisted neck dissections via a transaxillary and retroauricular ("TARA") approach in head and neck cancer: preliminary results. Ann Surg Oncol 2012; 19 : 1009-1017

[81] Kitagawa M, Dokko D, Okamura AM et al. Effect of sensory substitution on suture-manipulation forces for robotic surgical systems. J Thorac Cardiovasc Surg 2005; 129: 151-158

[82] Koliakos N, Denaeyer G, Willemsen P et al. Failure of a robotic arm during da Vinci prostatectomy: a case report. J Robot Surg 2008; 2: 95-96

[83] Kraft M, Fostiropoulos K, Gurtler N et al. Value of narrow band imaging in the early diagnosis of laryngeal cancer. Head Neck 2016; 38: $15-20$

[84] Kraft M, Mende S, Arnoux A et al. Anatomical landmarks for endosonography of the larynx. Head Neck 2010; 32: 326-332

[85] Krishnan G, Krishnan S. Transoral Robotic Surgery Total Laryngectomy: Evaluation of Functional and Survival Outcomes in a Retrospective Case Series at a Single Institution. ORL J Otorhinolaryngol Relat Spec 2017; 79: 191-201

[86] Kristin J, Kolmer A, Kraus P et al. Development of a new endoscope holder for head and neck surgery - from the technical design concept to implementation. Eur Arch Otorhinolaryngol 2015; 272: 1239-1244

[87] Kubik M, Mandal R, Albergotti W et al. Effect of transcervical arteria ligation on the severity of postoperative hemorrhage after transoral robotic surgery. Head Neck 2017; 39: 1510-1515

[88] Kucur C, Durmus K, Dziegielewski PT et al. Transoral robot-assisted carbon dioxide laser surgery for hypopharyngeal cancer. Head Neck 2015; 37: 743-745

[89] Kupferman ME, Demonte F, Levine $\mathrm{N}$ et al. Feasibility of a robotic surgical approach to reconstruct the skull base. Skull Base $2011 ; 21$ : 79-82

[90] Kuppersmith RB, Holsinger FC. Robotic thyroid surgery: an initial experience with North American patients. Laryngoscope 2011; 121 : 521-526

[91] Laccourreye O, Malinvaud D, Garcia D et al. Postoperative hemorrhage after transoral oropharyngectomy for cancer of the lateral oropharynx. Ann Otol Rhinol Laryngol 2015; 124: 361-367

[92] Lallemant B, Chambon G, Galy-Bernadoy C.et al. Transaxillary robotic thyroid surgery: a preliminary European experience. Eur Thyroid J 2013; 2: 110-115

[93] Lallemant B, Chambon G, Garrel R et al. Transoral robotic surgery for the treatment of T1-T2 carcinoma of the larynx: preliminary study. Laryngoscope 2013; 123: 2485-2490

[94] Lang S, Mattheis S, Hasskamp P et al. A european multicenter study evaluating the flex robotic system in transoral robotic surgery. Laryngoscope 2016; 127: 391-395 doi: 10.1002/lary.26358. Epub 2016 Oct 26

[95] Lawson G, Mendelsohn AH, Van Der Vorst S et al. Transoral robotic surgery total laryngectomy. Laryngoscope 2013; 123: 193-196
[96] Lee HS, Kim D, Lee SY et al. Robot-assisted versus endoscopic submandibular gland resection via retroauricular approach: a prospective nonrandomized study. Br J Oral Maxillofac Surg 2014; 52: 179-184

[97] Lee HS, Kim WS, Hong HJ et al. Robot-assisted Supraomohyoid neck dissection via a modified face-lift or retroauricular approach in early-stage cN0 squamous cell carcinoma of the oral cavity: a comparative study with conventional technique. Ann Surg Oncol 2012; 19: 3871-3878

[98] Lee HY, You JY, Woo SU et al. Transoral periosteal thyroidectomy: cadaver to human. Surg Endosc 2015; 29: 898-904

[99] Lee J, Kang SW, Jung J] et al. Multicenter study of robotic thyroidectomy: short- term postoperative outcomes and surgeon ergonomic considerations. Ann Surg Oncol 2011; 18: 2538-2547

[100] Lee J, Yun JH, Choi UJ et al. Robotic versus Endoscopic Thyroidectomy for Thyroid Cancers: A Multi-Institutional Analysis of Early Postoperative Outcomes and Surgical Learning Curves. J Oncol 2012; 2012: 734541

[101] Lee JY, Lega B, Bhowmick D et al. Da Vinci Robot-assisted transoral odontoidectomy for basilar invagination. ORL J Otorhinolaryngol Relat Spec 2010; 72: 91-95

[102] Lee S, Kim HY, Lee CR et al. A prospective comparison of patient body image after robotic thyroidectomy and conventional open thyroidectomy in patients with papillary thyroid carcinoma. Surgery 2014; 156: 117-125

[103] Lee SY, Park YM, Byeon HK et al. Comparison of oncologic and functional outcomes after transoral robotic lateral oropharyngectomy versus conventional surgery for T1 to T3 tonsillar cancer. Head Neck 2014; 36: 1138-1145

[104] Lim SC, Lee HK, Park J. Role of combined tactile and kinesthetic feedback in minimally invasive surgery (ePub!). Int J Med Robot 2014 Oct 18. doi: 10.1002/rcs.1625. [Epub ahead of print]

[105] Linos D, Kiriakopoulos A, Petralias A. Patient attitudes toward transaxillary robot-assisted thyroidectomy. World J Surg 2013; 37: 1959-1965

[106] Lira RB, Chulam TC, De Carvalho GB et al. Retroauricular endoscopic and robotic versus conventional neck dissection for oral cancer (ePub). J Robot Surg 2017 May 4. doi: 10.1007/s11701-017-0706-0. [Epub ahead of print]

[107] Lobe TE, Wright SK, Irish MS. Novel uses of surgical robotics in head and neck surgery. J Laparoendosc Adv Surg Tech A 2005; 15 : 647-652

[108] Lorincz BB, Busch C], Mockelmann N et al. Feasibility and safety of transoral robotic surgery (TORS) for early hypopharyngeal cancer: a subset analysis of the Hamburg University TORS-trial. Eur Arch Otorhinolaryngol 2015; 272: 2993-2998

[109] Lorincz BB, Mockelmann N, Busch C] et al. Two-Year Survival Analysis of 50 Consecutive Head and Neck Cancer Patients Treated with Transoral Robotic Surgery in a Single European Centre. Ann Surg Oncol 2015; 22: (Suppl 3): 1028-1033

[110] Ly HH, Tanaka Y, Fukuda T et al. Grasper having tactile sensing function using acoustic reflection for laparoscopic surgery. Int ] Comput Assist Radiol Surg 2017; 12: 1333-1343

[111] Marescaux J, Leroy J, Gagner $M$ et al. Transatlantic robot-assisted telesurgery. Nature 2001; 413: 379-380

[112] Mattheis S, Hoffmann TK, Schuler PJ et al. [The use of a flexible CO2-laser fiber in transoral robotic surgery (TORS)]. Laryngorhinootologie 2014; 93: 95-99

[113] Mattheis S, Mandapathil M, Rothmeier N et al. [Transoral robotic surgery for head and neck tumors: a series of 17 patients]. Laryngorhinootologie 2012; 91: 768-773 
[114] Mattos LS, Deshpande N, Barresi G et al. A novel computerized surgeon- machine interface for robot-assisted laser phonomicrosurgery. Laryngoscope 2014; 124: 1887-1894

[115] Mccool RR, Warren FM, Wiggins RH $3^{\text {rd }}$ et al. Robotic surgery of the infratemporal fossa utilizing novel suprahyoid port. Laryngoscope 2010; 120: 1738-1743

[116] Meccariello G, Cammaroto G, Montevecchi F et al. Transoral robotic surgery for the management of obstructive sleep apnea: a systematic review and meta-analysis. Eur Arch Otorhinolaryngol 2017; 274: 647-653

[117] Meccariello G, Faedi F, Alghamdi S et al. An experimental study about haptic feedback in robotic surgery: may visual feedback substitute tactile feedback? J Robot Surg 2016; 10: 57-61

[118] Mendelsohn AH, Remacle M, Van Der Vorst S et al. Outcomes following transoral robotic surgery: supraglottic laryngectomy. Laryngoscope 2013; 123: 208-214

[119] Mercante G, Ruscito P, Pellini R et al. Transoral robotic surgery (TORS) for tongue base tumours. Acta Otorhinolaryngol Ital 2013; 33: 230-235

[120] Meulemans J, Delaere P, Vander Poorten V. Early experience in transoral robotic surgery (TORS) for non-oropharyngeal head and neck malignancies: a review of functional and oncologic outcomes. B-ENT 2015; Suppl 24: 21-31

[121] Meulemans ], Vanclooster C, Vauterin T et al. Up-front and Salvage Transoral Robotic Surgery for Head and Neck Cancer: A Belgian Multicenter Retrospective Case Series. Front Oncol 2017; 7: 15

[122] Moore EJ, Janus J, Kasperbauer J. Transoral robotic surgery of the oropharynx: Clinical and anatomic considerations. Clin Anat 2012; 25: $135-141$

[123] Moore E], Van Abel KM, Olsen KD. Transoral robotic surgery in the seated position: Rethinking our operative approach. Laryngoscope 2017; 127: 122-126

[124] Morimoto TK, Greer JD, Hsieh MH et al. Surgeon Design Interface for Patient- Specific Concentric Tube Robots. Proc IEEE RAS EMBS Int Conf Biomed Robot Biomechatron 2016; 2016: 41-48

[125] Mukhija VK, Sung CK, Desai SC et al. Transoral robotic assisted free flap reconstruction. Otolaryngol Head Neck Surg 2009; 140: 124-125

[126] Nathan CO, Chakradeo V, Malhotra K et al. The voice-controlled robotic assist scope holder AESOP for the endoscopic approach to the sella. Skull Base 2006; 16: 123-131

[127] Nichols AC, Yoo J, Hammond JA et al. Early-stage squamous cell carcinoma of the oropharynx: radiotherapy vs. trans-oral robotic surgery (ORATOR) - study protocol for a randomized phase II trial. BMC Cancer 2013; 13: 133

[128] Noh Y, Bimbo J, Sareh S et al. Multi-Axis Force/Torque Sensor Based on Simply-Supported Beam and Optoelectronics. Sensors (Basel) 2016; 16: pii: E1936

[129] O'malley BW Jr., Quon H, Leonhardt FD et al. Transoral robotic surgery for parapharyngeal space tumors. ORL J Otorhinolaryngol Relat Spec 2010; 72: 332-336

[130] O'malley BW Jr., Weinstein GS. Robotic anterior and midline skull base surgery: preclinical investigations. Int J Radiat Oncol Biol Phys 2007; 69: S 125-S 128

[131] O'malley BW Jr., Weinstein GS, Snyder W et al. Transoral robotic surgery (TORS) for base of tongue neoplasms. Laryngoscope 2006; 116: $1465-1472$

[132] Okamura AM. Haptic feedback in robot-assisted minimally invasive surgery. Curr Opin Urol 2009; 19: 102-107

[133] Olds K, Hillel A, Kriss J et al. A robotic assistant for trans-oral surgery: the robotic endo-laryngeal flexible (Robo-ELF) scope. J Robot Surg 2012; 6: 13-18
[134] Olds K, Hillel AT, Cha E et al. Robotic endolaryngeal flexible (Robo-ELF) scope: a preclinical feasibility study. Laryngoscope 2011; 121: $2371-2374$

[135] Olsen SM, Moore E], Koch CA et al. Transoral robotic surgery for supraglottic squamous cell carcinoma. Am J Otolaryngol 2012; 33 : 379-384

[136] Ozer E, Durmus K, Carrau RL et al. Applications of transoral, transcervical, transnasal, and transpalatal corridors for robotic surgery of the skull base. Laryngoscope 2013; 123: 2176-2179

[137] Ozer E, Waltonen J. Transoral robotic nasopharyngectomy: a novel approach for nasopharyngeal lesions. Laryngoscope 2008; 118 : 1613-1616

[138] Pacchierotti C, Prattichizzo D, Kuchenbecker KJ. Cutaneous Feedback of Fingertip Deformation and Vibration for Palpation in Robotic Surgery. IEEE Trans Biomed Eng 2016; 63: 278-287

[139] Park YM, Byeon HK, Chung HP et al. Comparison of treatment outcomes after transoral robotic surgery and supraglottic partial laryngectomy: Our experience with seventeen and seventeen patients respectively. Clin Otolaryngol 2013; 38: 270-274

[140] Park YM, Byeon HK, Chung HP et al. Comparison study of transoral robotic surgery and radical open surgery for hypopharyngeal cancer. Acta Otolaryngol 2013; 133: 641-648

[141] Park YM, Jung CM, Cha D et al. The long-term oncological and functional outcomes of transoral robotic surgery in patients with hypopharyngeal cancer. Oral Oncol 2017; 71: 138-143

[142] Park YM, Lee WJ, Yun IS et al. Free flap reconstruction after robot-assisted neck dissection via a modified face-lift or retroauricular approach. Ann Surg Oncol 2013; 20: 891-898

[143] Pollei TR, Hinni ML, Moore EJ et al. Analysis of postoperative bleeding and risk factors in transoral surgery of the oropharynx. JAMA Otolaryngol Head Neck Surg 2013; 139: 1212-1218

[144] Rangarajan S, Hachem RA, Ozer E et al. Robotics in Sinus and Skull Base Surgery. Otolaryngol Clin North Am 2017; 50: 633-641

[145] Razafindranaly V, Lallemant B, Aubry K et al. Clinical outcomes with transoral robotic surgery for supraglottic squamous cell carcinoma: Experience of a French evaluation cooperative subgroup of GETTEC. Head Neck 2016; 38: (Suppl 1): E1097-E1101

[146] Reiley CE, Akinbiyi T, Burschka D et al. Effects of visual force feedback on robot-assisted surgical task performance. J Thorac Cardiovasc Surg 2008; 135: 196-202

[147] Richmon JD. Transoral palate-sparing nasopharyngectomy with the Flex(R) System: preclinical study. Laryngoscope 2015; 125: 318-322

[148] Richmon JD, Holsinger FC, Kandil E et al. Transoral robotic-assisted thyroidectomy with central neck dissection: preclinical cadaver feasibility study and proposed surgical technique. J Robot Surg 2011; 5: $279-282$

[149] Richmon JD, Kim HY. Transoral robotic thyroidectomy (TORT): procedures and outcomes. Gland Surg 2017; 6: 285-289

[150] Richmon JD, Pattani KM, Benhidjeb T et al. Transoral robotic-assisted thyroidectomy: a preclinical feasibility study in 2 cadavers. Head Neck 2011; 33: 330-333

[151] Rodin D, Caulley L, Burger E et al. Cost-Effectiveness Analysis of Radiation Therapy Versus Transoral Robotic Surgery for Oropharyngeal Squamous Cell Carcinoma. Int J Radiat Oncol Biol Phys 2017; 97: 709-717

[152] Rudmik L, An W, Livingstone D et al. Making a case for high-volume robotic surgery centers: A cost-effectiveness analysis of transoral robotic surgery. J Surg Oncol 2015; 112: 155-163

[153] Russell JO, Clark J, Noureldine SI et al. Transoral thyroidectomy and parathyroidectomy - A North American series of robotic and endoscopic transoral approaches to the central neck. Oral Oncol 2017; 71: 75-80 
[154] Saccomandi P, Schena E, Oddo CM et al. Microfabricated tactile sensors for biomedical applications: a review. Biosensors (Basel) 2014; 4: 422-448

[155] Schneider JS, Burgner J, Webster RJ $3^{\text {rd }}$ et al. Robotic surgery for the sinuses and skull base: what are the possibilities and what are the obstacles? Curr Opin Otolaryngol Head Neck Surg 2013; 21: 11-16

[156] Schoob A, Kundrat D, Kahrs LA et al. Comparative study on surface reconstruction accuracy of stereo imaging devices for microsurgery. Int J Comput Assist Radiol Surg 2016; 11: 145-156

[157] Schoob A, Laves MH, Kahrs LA et al. Soft tissue motion tracking with application to tablet-based incision planning in laser surgery. Int J Comput Assist Radiol Surg 2016; 11: 2325-2337

[158] Schuler PJ, Duvvuri U, Friedrich DT et al. First use of a computerassisted operator-controlled flexible endoscope for transoral surgery. Laryngoscope 2015; 125: 645-648

[159] Schuler PJ, Hoffmann TK, Duvvuri U et al. Demonstration of nasopharyngeal surgery with a single port operator-controlled flexible endoscope system. Head Neck 2016; 38: 370-374

[160] Schuler PJ, Hoffmann TK, Veit JA et al. Hybrid procedure for total laryngectomy with a flexible robot-assisted surgical system. Int J Med Robot 2016; 13. doi: 10.1002/rcs.1749. Epub 2016 May 16.

[161] Schuler PJ, Scheithauer M, Rotter $\mathrm{N}$ et al. A single-port operatorcontrolled flexible endoscope system for endoscopic skull base surgery. HNO 2015; 63: 189-194

[162] Sher DJ, Fidler MJ, Tishler RB et al. Cost-Effectiveness Analysis of Chemoradiation Therapy Versus Transoral Robotic Surgery for Human Papillomavirus- Associated, Clinical N2 Oropharyngeal Cancer. Int J Radiat Oncol Biol Phys 2016; 94: 512-522

[163] Smith RV, Schiff BA, Sarta C et al. Transoral robotic total laryngectomy. Laryngoscope 2013; 123: 678-682

[164] Solares CA, Strome M. Transoral robot-assisted CO2 laser supraglottic laryngectomy: experimental and clinical data. Laryngoscope 2007; 117: $817-820$

[165] Song CM, Cho YH, Ji YB et al. Comparison of a gasless unilateral axillo-breast and axillary approach in robotic thyroidectomy. Surg Endosc 2013; 27: 3769-3775

[166] Song CM, Yun BR, Ji YB et al. Long-Term Voice Outcomes After Robotic Thyroidectomy. World J Surg 2016; 40: 110-116

[167] Song HG, Yun IS, Lee W] et al. Robot-assisted free flap in head and neck reconstruction. Arch Plast Surg 2013; 40: 353-358

[168] Sreenath SB, Rawal RB, Zanation AM. The combined endonasal and transoral approach for the management of skull base and nasopharyngeal pathology: a case series. Neurosurg Focus 2014; 37: E2

[169] Stock K, Diebolder R, Hausladen F et al. Primary investigations on the potential of a novel diode pumped Er: YAG laser system for bone surgery. Photonic Therapeutics and Diagnostics IX, Proceedings of the SPIE 85656, 85656D. 2013

[170] Stock K, Stegmayer T, Graser R et al. Comparison of different focusing fiber tips for improved oral diode laser surgery. Lasers Surg Med 2012; 44: 815-823

[171] Strauss G, Hofer M, Kehrt S et al. [Manipulator assisted endoscope guidance in functional endoscopic sinus surgery: proof of concept]. HNO 2007; 55: 177-184

[172] Tae K, Ji YB, Song CM et al. Robotic selective neck dissection using a gasless postauricular facelift approach for early head and neck cancer: technical feasibility and safety. J Laparoendosc Adv Surg Tech A 2013; 23: 240-245

[173] Tang B, Hanna GB, Cuschieri A. Analysis of errors enacted by surgical trainees during skills training courses. Surgery 2005; 138: 14-20

[174] Terris DJ, Singer MC. Qualitative and quantitative differences between 2 robotic thyroidectomy techniques. Otolaryngol Head Neck Surg 2012; 147: 20-25
[175] Thaler ER, Rassekh CH, Lee JM et al. Outcomes for multilevel surgery for sleep apnea: Obstructive sleep apnea, transoral robotic surgery, and uvulopalatopharyngoplasty. Laryngoscope 2016; 126: 266-269

[176] Tibbetts KM, Tan M. Role of Advanced Laryngeal Imaging in Glottic Cancer: Early Detection and Evaluation of Glottic Neoplasms. Otolaryngol Clin North Am 2015; 48: 565-584

[177] Trevillot V, Garrel R, Dombre E et al. Robotic endoscopic sinus and skull base surgery: review of the literature and future prospects. Eur Ann Otorhinolaryngol Head Neck Dis 2013; 130: 201-207

[178] Troob S, Givi B, Hodgson M et al. Transoral robotic retropharyngeal node dissection in oropharyngeal squamous cell carcinoma: Patterns of metastasis and functional outcomes. Head Neck 2017; 39: 1969-1975

[179] Tsang RK, Ho WK, Wei WI et al. Transoral robotic assisted nasopharyngectomy via a lateral palatal flap approach. Laryngoscope 2013; 123: $2180-2183$

[180] Tsang RK, Holsinger FC. Transoral endoscopic nasopharyngectomy with a flexible next-generation robotic surgical system. Laryngoscope 2016; 126: 2257-2262

[181] Tsang RK, To VS, Ho AC et al. Early results of robotic assisted nasopharyngectomy for recurrent nasopharyngeal carcinoma. Head Neck 2015; 37: 788-793

[182] Van Der Meijden OA, Schijven MP. The value of haptic feedback in conventional and robot-assisted minimal invasive surgery and virtual reality training: a current review. Surg Endosc 2009; 23: 1180-1190

[183] Vicini C, Montevecchi F, Campanini A et al. Clinical outcomes and complications associated with TORS for OSAHS: a benchmark for evaluating an emerging surgical technology in a targeted application for benign disease. ORL J Otorhinolaryngol Relat Spec 2014; 76: 63-69

[184] Wagner OJ, Hagen M, Kurmann A et al. Three-dimensional vision enhances task performance independently of the surgical method. Surg Endosc 2012; 26: 2961-2968

[185] Wang C, Kundaria S, Fernandez-Miranda J et al. A description of the anatomy of the glossopharyngeal nerve as encountered in transoral surgery. Laryngoscope 2016; 126: 2010-2015

[186] Wang CC, Liu SA, Wu SH et al. Transoral robotic surgery for early glottic carcinoma involving anterior commissure: Preliminary reports. Head Neck 2016; 38: 913-918

[187] Wang CC, Liu SA, Wu SH et al. Transoral robotic surgery for early T classification hypopharyngeal cancer. Head Neck 2016; 38: 857-862

[188] Wei WI, Chan JY, Ng RW et al. Surgical salvage of persistent or recurrent nasopharyngeal carcinoma with maxillary swing approach - Critical appraisal after 2 decades. Head Neck 2011; 33: 969-975

[189] Wei WI, Ho WK. Transoral robotic resection of recurrent nasopharyngeal carcinoma. Laryngoscope 2010; 120: 2011-2014

[190] Wei WI, Kwong DL. Recurrent nasopharyngeal carcinoma: surgical salvage vs. additional chemoradiation. Curr Opin Otolaryngol Head Neck Surg 2011; 19: 82-86

[191] Weinstein GS, O'malley BW Jr., Hockstein NG. Transoral robotic surgery: supraglottic laryngectomy in a canine model. Laryngoscope 2005; 115: 1315-1319

[192] Weinstein GS, O'malley BW Jr., Magnuson JS et al. Transoral robotic surgery: a multicenter study to assess feasibility, safety, and surgical margins. Laryngoscope 2012; 122: 1701-1707

[193] Weinstein GS, O'malley BW Jr., Snyder W et al. Transoral robotic surgery: supraglottic partial laryngectomy. Ann Otol Rhinol Laryngol 2007; 116: 19-23

[194] Weinstein GS, O'malley BW Jr., Snyder W et al. Transoral robotic surgery: radical tonsillectomy. Arch Otolaryngol Head Neck Surg 2007; 133: 1220-1226 
[195] Wottawa CR, Genovese B, Nowroozi BN et al. Evaluating tactile feedback in robotic surgery for potential clinical application using an animal model. Surg Endosc 2016; 30: 3198-3209

[196] Wu L, Song S, Wu K et al. Development of a compact continuum tubular robotic system for nasopharyngeal biopsy. Med Biol Eng Comput 2017; 55: 403-417

[197] Yeh DH, Tam S, Fung K et al. Transoral robotic surgery vs. radiotherapy for management of oropharyngeal squamous cell carcinoma - A systematic review of the literature. Eur J Surg Oncol 2015; 41: 1603-1614
[198] Yin Tsang RK, Ho WK, Wei WI. Combined transnasal endoscopic and transoral robotic resection of recurrent nasopharyngeal carcinoma. Head Neck 2012; 34: 1190-1193

[199] Zenga J, Suko J, Kallogjeri D et al. Postoperative hemorrhage and hospital revisit after transoral robotic surgery. Laryngoscope 2017; 127: $2287-2292$

[200] Zhou S, Zhang C, Li D. Approaches of robot-assisted neck dissection for head and neck cancer: a review. Oral Surg Oral Med Oral Pathol Oral Radiol 2016; 121: 353-359 